Les disparités géographiques de la mortalité dans le Montréal métropolitain, 1984-1988 : étude écologique des liens avec les conditions sociales, économiques et culturelles SPATIAL MORTALITY DISPARITIES IN THE METROPOLITAN REGION OF MONTREAL, 1984-1988: AN ECOLOGICAL ANALYSIS OF THE ROLE OF SOCIAL, ECONOMIC AND CULTURAL FACTORS DESNIVELES GEOGRAFICOS DE MORTAUDAD EN LA REGION METROPOLITANA DE MONTREAL, 1984-1988: ANALISIS ECOLOGICO DE LA FUNCION DE LAS CONDICIONES SOCIALES, ECONOMICAS Y CULTURALES

Robert Choinière

Volume 20, numéro 1, printemps 1991

URI : https://id.erudit.org/iderudit/010068ar

DOI : https://doi.org/10.7202/010068ar

Aller au sommaire du numéro

Éditeur(s)

Association des démographes du Québec

ISSN

0380-1721 (imprimé)

1705-1495 (numérique)

Découvrir la revue

Citer cet article

Choinière, R. (1991). Les disparités géographiques de la mortalité dans le Montréal métropolitain, 1984-1988 : étude écologique des liens avec les conditions sociales, économiques et culturelles. Cahiers québécois de démographie, 20(1), 115-144. https://doi.org/10.7202/010068ar

\section{Résumé de l'article}

Malgré une baisse notable des indices de mortalité dans la région de Montréal, l'on retrouve toujours à la fin des années 1980 des disparités géographiques importantes. Un écart de plus de dix ans en espérance de vie est observé entre certains quartiers, et cet écart n'a pas diminué durant les dix dernières années. L'étude écologique des liens entre la mortalité et différentes caractéristiques de la population à partir des méthodes de la régression linéaire montre que la proportion de personnes pauvres et le pourcentage d'immigrants combinés expliqueraient ensemble plus de $80 \%$ des variations géographiques de l'espérance de vie à Montréal. 
Cahiers québécois de démographie

Vol. 20, no 1, printemps 1991, p. 115-144

\title{
Les disparités géographiques de la mortalité dans le Montréal métropolitain, 1984-1988 : étude écologique des liens avec les conditions sociales, économiques et culturelles
}

\author{
Robert CHOINIËRE *
}

La population de la région de Montréal, comme celle de l'ensemble du Québec, a enregistré une baisse importante de sa mortalité depuis le début des années 1950. De 1951 à 1986, l'espérance de vie à la naissance est passée de 69,4 ans à 79,5 ans chez les femmes, et de 64,5 ans à 72,5 ans chez les hommes. L'évolution de la mortalité infantile a été encore plus spectaculaire. De 32,0 pour mille au dêbut des années 1950 , le taux de mortalité infantlle a chuté à 7,0 pour mille en 1986 (tableau 1).

Les taux de mortalité infantile observés de nos jours dans la région montréalaise (7,0 p. 1000) et dans l'ensemble du Québec $(7,1$ p. 1000) se situent parmi les plus bas au monde et sont inférieurs aux valeurs obtenues pour l'Ontario (7,2 p. 1000) et pour l'ensemble du Canada (7,9 p. 1000). L'espérance de vie des Montréalais se compare aussi favorablement à celle des différents pays industrialisés. Dans ce dernier cas, cependant, la comparaison avantage l'Ontario et le Canada par rapport à Montréal et au Québec (tableau 2).

Malgré ces gains appréciables face à la mortalité, des écarts importants subsistent dans la région de Montréal selon le statut socio-économique et le lieu de résidence (Choinière, 1990b; Guillemette, 1983; Hoey, Wilkdns, Gagnon et O'Loughlin, 1987; O'Loughlin et Bolvin, 1987; Wilkins, 1979, 1980a, 1980b. $1980 \mathrm{c})$. Une étude récente sur l'évolution de la mortalité selon le

* Département de santé communautaire, Hôpital général de Montréal. L'auteur remercie Denis Roy pour ses commentaires constructifs. 
TABLEAU 1

Espérance de vie à la naissance selon le sexe et taux de mortalité infantile, région de Montréal, 1951 à 1986

\begin{tabular}{lcccc}
\hline & \multicolumn{2}{c}{$\begin{array}{c}\text { Espérance } \\
\text { de vie } \\
\text { à la } \\
\text { naissance }\end{array}$} & & \multicolumn{2}{c}{$\begin{array}{c}\text { Taux de morta- } \\
\text { lité infantile } \\
\text { (pour 1000 } \\
\text { naissances) }\end{array}$} \\
\cline { 2 - 3 } Période & Hommes & Femmes & Pêriode & Taux \\
\hline $1950-1952$ & 64,5 & 69,4 & $1951-1955$ & 32,0 \\
$1955-1957$ & 65,9 & 71,2 & $1956-1960$ & 26,7 \\
$1960-1962$ & 67,5 & 73,3 & $1961-1965$ & 23,2 \\
$1965-1967$ & 67,9 & 74,3 & $1966-1969$ & 20,8 \\
$1970-1972$ & 68,8 & 76,0 & $1970-1972$ & 16,9 \\
$1975-1977$ & 69,0 & 76,7 & $1975-1977$ & 12,0 \\
$1979-1981$ & 71,0 & 78,7 & $1979-1981$ & 9,0 \\
$1982-1984$ & 71,9 & 79,1 & $1982-1984$ & 7,9 \\
$1985-1987$ & 72,5 & 79,5 & $1985-1987$ & 7,0 \\
\hline
\end{tabular}

Sources : Ministère des Affaires sociales, 1970: Bureau de la statistique du Québec, 1972. 1988; MSSS, fichiers des naissances et des décès, 1979 à 1987; Wilkins, 1987; Choinière, 1990a.

a Pour les périodes 1950-1952 à 1965-1967, les espérances de vie ont été calculées pour l'ensemble de la région 06, comprenant la Rdve-Sud de Montréal et les Laurentides.

revenu dans les régions urbaines du Canada entre 1971 et 1986 conclut à une légère diminution des écarts d'espérance de vie entre les différents niveaux de revenu. Cependant, l'écart observé entre les quintiles extrêmes est plus de deux fois supérieur à l'écart enregistré entre les cinq régions du Canada (Wilkins, Adams et Brancker, 1989 : 166).

Dans la région de Montréal, les inégalitês de mortalité ne semblent pas diminuer dans le temps. De 1961 à 1976, les disparités entre zones défavorisées et zones privilégiées auraient même augmenté (Guillemette, 1983:44; Wilkins, 1980 c : 9). Les données les plus récentes montrent que l'espérance de vie à la naissance des résidants de Saint-Henri, l'un des quartiers les plus défavorisês de Montréal, est de 9 ans inférieure à celle des résidants de Westmount, l'une des municipalités les plus riches de la région métropolitaine, et cet écart est resté stable depuis 1976 (Choinière, 1990b; O'Loughlin et Bolvin, 1987; Wilkins et Adams, 1983).

Cet article a pour objectif d'examiner les disparités géographiques de la mortalité dans la région de Montréal durant la période 1984-1988, de comparer la situation avec celle de la 
TABLEAU 2

Espérance de vie à la naissance selon le sexe et taux de mortalité infantile (comparaisons internationales), autour de 1986

\begin{tabular}{|c|c|c|c|c|c|}
\hline & \multicolumn{3}{|c|}{$\begin{array}{l}\text { Espérance de vie } \\
\text { à la naissance }\end{array}$} & \multicolumn{2}{|c|}{$\begin{array}{l}\text { Taux de morta- } \\
\text { lité infantile } \\
\text { (pour } 1000 \\
\text { naissances) }\end{array}$} \\
\hline & Période & Hommes & Femmes & Période & Taux \\
\hline Région de Montréal & $1985-1987$ & 72,5 & 79,5 & $1985-1987$ & 7,0 \\
\hline Québec & $1985-1987$ & 71,9 & 79,4 & 1986 & 7,1 \\
\hline Ontario & $1985-1987$ & 73,5 & 79,8 & 1986 & 7,2 \\
\hline Canada & $1985-1987$ & 73,1 & 79,8 & 1986 & 7,9 \\
\hline États-Unis & 1986 & 71,3 & 78,3 & 1986 & 10,4 \\
\hline Japon & 1986 & 75,4 & 81,3 & 1985 & 5,5 \\
\hline Autriche & 1986 & 71,0 & 77,7 & 1987 & 9,8 \\
\hline Danemark & 1985 & 71,6 & 77.5 & 1985 & 7,9 \\
\hline Finlande & 1985 & 70,1 & 78,5 & 1986 & 6,5 \\
\hline France & 1986 & 71,5 & 79,6 & 1987 & 7,6 \\
\hline Islande & 1986 & 75,0 & 80,4 & 1986 & 5,4 \\
\hline Norvège & 1985 & 72,8 & 79,5 & 1986 & 7,8 \\
\hline Pays-Bas & 1985 & 73,1 & 79,7 & 1987 & 7,6 \\
\hline Suède & 1986 & 74,0 & 80,0 & 1986 & 5,9 \\
\hline Suisse & 1986 & 73,6 & 80,3 & 1986 & 6,8 \\
\hline
\end{tabular}

Sources : Monnier, 1988; tableau 4; Statistique Canada, 1990, 1988.

période 1979-1983 et d'étudier, selon une approche écologique, les relations entre la mortalité et certaines caractéristiques sociales, culturelles et économiques. Cette étude se démarque des travaux réalisés jusqu'à maintenant sur la mortalité différentielle à Montréal (Wilkins, 1979, 1980a, 1980c; Guillemette, 1983), qui utilisent un découpage en zones socio-économiquement homogènes afin de mesurer les disparités sociales face à la mort.

Nous retenons un découpage différent, qui englobe l'ensemble du Montréal métropolitain (île de Montréal et Laval) et comprend un plus grand nombre d'unites territoriales (40). L'approche employée est également différente. En premier lieu, nous identifions les disparitês géographiques, et nous tentons par la suite de les expliquer en étudiant les liens entre la mortalité et différentes caractéristiques de la population à partir des méthodes de la régression linéaire, en ne nous limitant pas au statut socio-économique. 


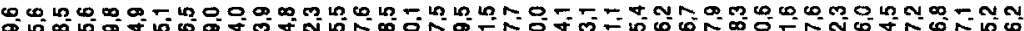
सं

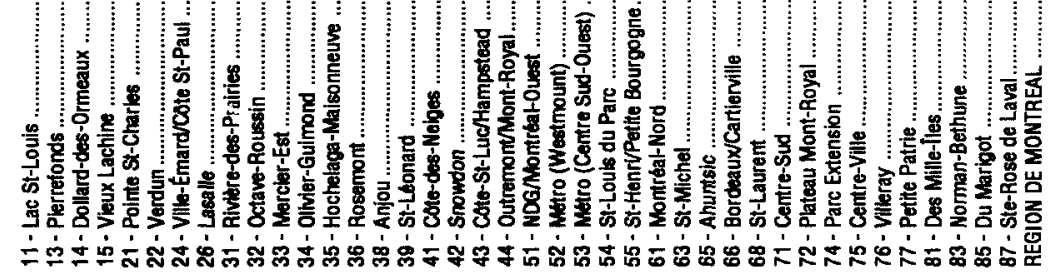

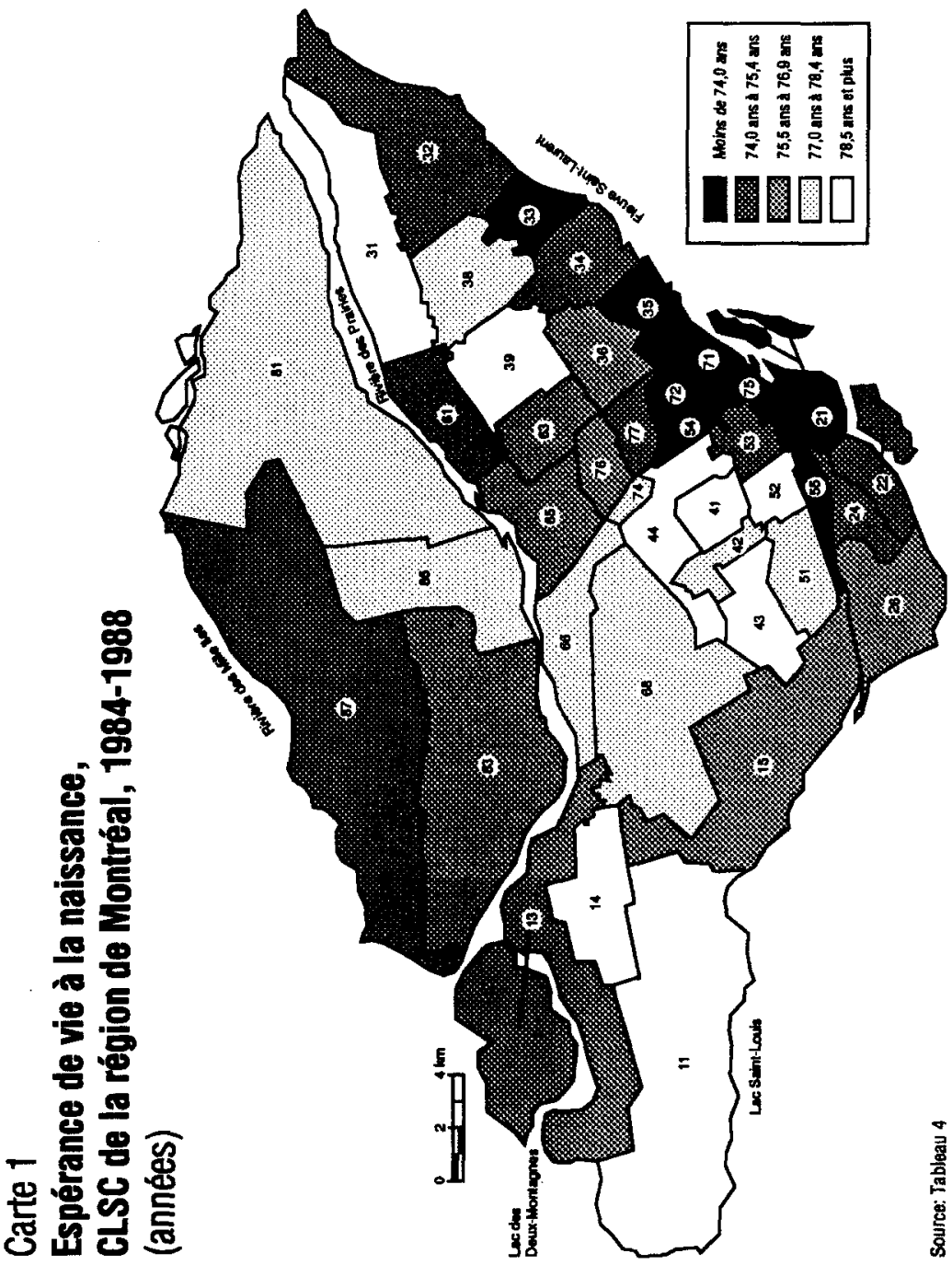




\section{METHODOLOGIE}

Les deux indicateurs retenus pour mesurer la mortalité sont l'espérance de vie à la naissance et le taux de mortalité infantile. Ces indicateurs ont été calculés pour chacun des territoires de centres locaux de services communautaires (CLSC) situés sur le territoire de la région socio-sanitaire du Montréal métropolitain (île de Montréal et Laval).

Les trente-neuf territoires de CLSC retenus correspondent à la définition des limites géographiques établie par le Conseil de la santé et des services sociaux de la région du Montréal métropolitain au mois d'octobre 1987. Ce découpage ne tient pas compte des CLSC qui ont été fusionnés à des fins administratives. Il ne correspond donc pas à celui qui est utilisé par le ministère de la Santé et des Services sociaux (MSSS), lequel retient trente-quatre territoires de CLSC.

Un quarantième territoire a été créé par la séparation du CLSC Métro en deux secteurs, Westmount et Centre Sud-Ouest.

Les quarante territoires ainsi obtenus correspondent à un découpage administratif de la région et ne constituent pas nécessairement des unités socio-économiquement homogènes. L'on peut cependant associer la plupart des CLSC à des quartiers (Pointe Saint-Charles, Saint-Michel, Saint-Henri, Rivière-des-Prairies, Côte-des-Neiges. Snowdon, Rosemont, Parc Extension, etc.) ou à des municipalités (Saint-Léonard, Dollard-des-Ormeaux, Anjou, Westmount, Montréal-Nord, etc.) (carte 1).

Une étude écologique des liens entre la mortalité et les conditions sociales, culturelles et économiques a été faite à partir des méthodes de l'analyse de régression linéaire, l'unité d'observation étant le territoire de CLSC. L'approche êcologique présente plusieurs limites pour l'étude des liens entre phénomènes. Les liens entre les variables observées à partir de données relatives à des territoires ne permettent pas d'inférer des liens à l'échelle des individus. En thêorie, le degré d'association écologique peut être plus fort ou plus faible que le degré d'association observé au niveau des individus. En pratique, la force de la relation obtenue par l'analyse écologique exagère généralement l'ampleur de la relation réelle (Morgenstern, 1982; Last, 1988; Langbein et Lichtman, 1988). Malgré leurs limites, les études écologiques sont utiles pour décrire les disparités dans la population et identifier les liens possibles entre phénomènes (Rothman, 1986). 
Les deux indicateurs de mortalité retenus représentent les variables dépendantes, et les caractéristiques sociales, économiques et culturelles, les variables indépendantes. Nous avons retenu huit variables indépendantes; chacune correspond à un champ particulier de l'environnement social :

\section{CHAMP}

Structure par âge et par sexe

Caractéristiques reliêes à la naissance

Caractéristiques

ethno-culturelles

Structure de la famille

Scolaritê

Population active

Profession

Revenu

\section{VARLABLES INDÉPENDANTES}

Rapport de dépendance : (Population de moins de 15 ans + population de 65 ans et plus)/Population de 15-64 ans

Proportion de naissances vivantes de faible poids (moins de 2500 grammes)

Proportion d'immigrants

Proportion de familles monoparentales (parmi l'ensemble des familles avec enfant[s])

Proportion de personnes de 15 ans et plus ayant moins de 9 ans de scolarité

Taux d'inoccupation (proportion de la population de 15 ans et plus n'ayant pas d'emploil)

Proportion de la population de 15 ans et plus occupée ayant un emploi dans les secteurs primaire ou secondaire

Proportion de personnes pauvres (sous le seuil de faible revenu)

Le tableau 3 présente les valeurs observées en 1986 pour chacune de ces huit variables indeppendantes dans chacun des quarante CLSC de la région de Montréal.

Les effectifs de population utilisés dans le calcul de l'espérance de vie à la naissance ainsi que les données socioéconomiques proviennent de totalisations spéciales du recensement (Choinière, 1990a; Wilkins, 1987). Les données sur les naissances et les décès ont été fournies par le ministère de la Santé et des Services sociaux. Les espérances de vie à la nais- 
TABLEAU 3

Résumé des variables indépendantes retenues, CLSC, région de Montréal, $1986^{\text {a }}$

\begin{tabular}{|c|c|c|c|c|c|c|c|c|}
\hline Territoires & 1 & 2 & 3 & 4 & 5 & 6 & 7 & 8 \\
\hline 11 - Lac St-Louis & 7,2 & 21,6 & 36,9 & 6,2 & 13,2 & 4.4 & 12.1 & 45.7 \\
\hline 13 - Pierrefonds & 12.6 & 17,8 & 36.1 & 10,5 & 17,0 & 5,6 & 22,5 & 41,7 \\
\hline 14 - Dollard-des-Ormeaux & 9,3 & 28,4 & 32,4 & 6,2 & 13,2 & 5,6 & 14,8 & 41,8 \\
\hline 15 - Vieux Lachine & 20,7 & 13,0 & 44,7 & 20,2 & 27,6 & 6.7 & 27,7 & 43,4 \\
\hline 21 - Pointe St-Charles & 52,0 & 5.3 & 63.0 & 40,0 & 45,1 & 8.9 & $\mathbf{3 7 , 8}$ & 44,6 \\
\hline 22 - Verdun & 29,6 & 8,2 & 49.1 & 26,3 & 37,0 & 7,1 & 26,1 & 41,1 \\
\hline 24 - Ville-Émard-Côte St-Paul & 28,5 & 10,6 & 49.1 & 33,7 & 28.6 & 7.5 & 33.6 & 40.1 \\
\hline 26 - Lasalle & 19,5 & 19,1 & 41.9 & 22,1 & 24,7 & 6.8 & 29,5 & 35,8 \\
\hline 31 - Rivière- & 20,0 & 27,1 & $\mathbf{3 8 . 8}$ & 29,8 & 14,5 & 5,6 & 41,6 & 42.2 \\
\hline 32- Octav & 19,3 & 3,6 & 41.7 & 23,2 & 22,1 & 6,7 & 34,3 & 37,6 \\
\hline 33-Mercier-Est & 21,4 & 5,5 & 44,9 & 25,9 & 28,7 & 7.0 & 29,6 & 37,6 \\
\hline 34 - Olivier-Guimond & 20.0 & 11.3 & 41.8 & 25.8 & 28,0 & 6.8 & 27.9 & 33.7 \\
\hline $35-$ Hoch & 40,3 & 5,4 & 54,8 & 35,1 & 44,0 & 8,5 & 34,6 & 39,5 \\
\hline $36-\operatorname{Ros}$ & 29,4 & 12,7 & 50,5 & 29,3 & 36,0 & $\mathbf{7 . 4}$ & 29,0 & 41,3 \\
\hline 38 - Anjou & 17.9 & 11.8 & 38.2 & 20,0 & 26,7 & 5.8 & 26.6 & 33,5 \\
\hline 39 - St-Léonard & 19,3 & 30,5 & 40,0 & 29,2 & 22,8 & 6,0 & 33,9 & 33,2 \\
\hline des-Neiges & 33,3 & 41,0 & 43,4 & 14,0 & 35,3 & 6.7 & 16.4 & 41.6 \\
\hline 42 - Snowdon & 33.1 & 53.1 & 48.1 & 19,4 & 32.3 & 5,9 & 21,8 & 51.9 \\
\hline 43 - Côte & 15,7 & 37,1 & 45,1 & 14,4 & 20.0 & 6,0 & 10,5 & 61.7 \\
\hline 44 - Outremont-M & 12,7 & 25,0 & 40,2 & 8,2 & 24,3 & 5,2 & 8,5 & 47,8 \\
\hline 51 - NDG-Montréal-Ouest & 23,8 & 31.5 & 42,7 & 13.1 & 31,4 & 6.1 & 14,4 & 45,8 \\
\hline 52 - Métro (Westmount) & 10,3 & 26,9 & 38,4 & 4,3 & 24,6 & 4,0 & 4,8 & 45,5 \\
\hline 53 - Métro & 31,3 & 33,0 & 35,1 & 8.9 & 39,7 & 6.2 & 9,6 & 25,8 \\
\hline $54-$ St-Lo & 42.0 & 38.0 & 45.2 & 29,0 & $\mathbf{3 3 , 4}$ & $\mathbf{5 . 7}$ & 27,0 & 33.4 \\
\hline $55-\mathrm{St}-\mathrm{He}$ & 46,6 & 12,6 & 57,2 & $\mathbf{3 4 , 5}$ & 45,5 & 8,8 & 30,8 & 41,4 \\
\hline 61 - Montréal-Nord & 28,2 & 16,0 & 46,3 & 28,3 & 31,9 & 8,1 & 37,7 & 41,2 \\
\hline 63 - St-M & 32,9 & 28,5 & 48,4 & 35,8 & 29,6 & 7.9 & 45.5 & 39.0 \\
\hline $65-$ Ahu & 21.7 & 17.6 & 43,2 & 22,6 & 28.0 & 6.2 & 25.5 & 39,0 \\
\hline 66 - Bordeaux-Cartierville & 22,4 & 32,6 & 43,6 & 18,5 & 23,5 & 5,5 & 23,8 & 39,1 \\
\hline 68 - St-Laurent & 22,9 & 35,0 & 42,5 & 17,0 & 27.4 & 5,3 & 21.8 & 43,0 \\
\hline 71 - Centre-Sud & 48.0 & 8.7 & 57,5 & 35.3 & 50.4 & 8,5 & 26,9 & 37,1 \\
\hline 72 - Plateau Mont-Ro & 39,0 & 8,8 & 47,2 & 25,4 & 46,6 & 8,4 & 22,5 & 31.7 \\
\hline 74 - Parc Extension & 50,1 & 57,8 & 53,1 & 42,3 & 24,2 & 6.8 & 43,9 & 43.9 \\
\hline 75 - Centre-Ville & 52,5 & 30.3 & 51.5 & 27,5 & 50,4 & 5,2 & 12.7 & 42,4 \\
\hline 76 - Villeray & 32,1 & 21.1 & 48,0 & 32,7 & 33,1 & 6,4 & $\mathbf{3 4 , 1}$ & 38,5 \\
\hline 77 - Petite Patrie & $\mathbf{3 8 . 4}$ & 16,0 & 51.1 & $\mathbf{3 3}, 0$ & $\mathbf{3 8 , 5}$ & 7.7 & 33,0 & 37,5 \\
\hline 81 -Des Mille-Îles & 12.7 & 8.0 & 37,6 & 15,6 & 15,6 & 6,0 & 27,2 & 33.3 \\
\hline 83 - Norman-Bethune & 18,4 & 19,7 & 40,2 & 18,8 & 19,4 & 5,9 & 27,3 & $\mathbf{3 7 . 7}$ \\
\hline 85 - Du Marigat & 18,1 & 7,9 & 39,2 & 18,1 & 22,4 & 6.7 & 26,5 & 35,5 \\
\hline 87 - Ste-Rose de I aval & 11,3 & 5.5 & 35,9 & 17,4 & 12.9 & 5.9 & 31.8 & 39,7 \\
\hline Région de Montréal & 24,5 & 20.1 & 44,8 & 22,4 & 26,8 & 6,5 & 26,4 & 39,6 \\
\hline
\end{tabular}

Source : Choinière, $1990 \mathrm{a}$.

a Titre des huit colonnes : 1) Personnes pauvres (\%); 2) Immigrants (\%); 3) Personnes inoccupées (\%); 4) Personnes ayant moins de 9 ans de scolarité; 5) Familles monoparentales (\%); 6) Naissances de faible poids; 7) Personnes de professions de secteur primaire ou tertiaire: 8) Rapport de dépendance. 
sance et les taux de mortalité infantile ont été calculés pour des périodes de cinq ans centrées sur les recensements de 1981 et de 1986.

\section{L'ESPERANCE DE VIE A LA NAISSANCE}

Le calcul de l'espérance de vie à la naissance à partir de tables de mortalitê du moment permet de comparer la longévité moyenne de différentes populations. La carte 1 présente, pour la période 1984-1988, l'espérance de vie à la naissance, sexes réunis, pour chacun des quarante territoires retenus; le tableau 4 présente cette même espérance de vie (sexes réunis) pour les périodes 1979-1983 et 1984-1988, en distinguant en outre selon le sexe dans le cas de cette dernière période.

L'espérance de vie à la naissance de la population montréalaise est de 76,2 ans. Les valeurs les plus faibles se retrouvent principalement dans la partie centre-sud de l'île de Montréal (en foncé sur la carte). Dans Pointe Saint-Charles (69.8 ans), Centre-Sud (70,6 ans), Saint-Henri-Petite Bourgogne ( 71,1 ans) et Plateau Mont-Royal (71,6 ans), la durée moyenne de la vie est inférieure à 72 ans. Les espérances de vie les plus èlevées sont observées au centre et à l'extrémité ouest de l'île de Montréal ainsi qu'à Saint-Léonard et à Rivière-des-Prairies. Les résidants de Westmount ( 80,0 ans), de Côte-des-Neiges (80,1 ans) et d'Outremont-Mont-Royal (81,5 ans) jouissent de longêvités moyennes égales ou supérieures à 80 ans.

L'espérance de vie à la naissance présente des écarts importants sur le territoire de la région de Montréal. L'on observe une différence de près de douze ans entre Pointe Saint-Charles et Outremont-Mont-Royal, les deux CLSC qui présentent les valeurs les plus extrêmes.

Pour l'ensemble de la population montréalaise, les femmes vivent en moyenne sept ans de plus que les hommes $(79,5$ ans contre 72,5 ans). L'écart entre les deux sexes tend cependant à diminuer depuis 1980; il étalt alors de 7.7 ans (tableau 1). L'espérance de vie présente les mêmes disparités gêographiques selon le sexe. Tant chez les hommes que chez les femmes, les valeurs les plus basses sont enregistrées dans Pointe SaintCharles (66,2 ans et 73,5 ans) et les plus hautes dans Outremont-Mont-Royal (78,5 ans et 83,6 ans). La différence entre ces deux territoires est cependant plus importante chez les hommes (12,3 ans) que chez les femmes (10,1 ans) (tableau 4). 
TABLEAU 4

Espérance de vie à la naissance, CLSC de la région de Montréal, 1979-1983 et 1984-1988 (en années)

\begin{tabular}{|c|c|c|c|c|c|c|}
\hline \multirow{3}{*}{ Territoires } & \multicolumn{3}{|c|}{$1984-1988$} & \multirow{3}{*}{$\begin{array}{l}1979- \\
1983 \\
\text { Total }\end{array}$} & \multirow{2}{*}{\multicolumn{2}{|c|}{$\begin{array}{c}\text { Variations } \\
1979-1983 / \\
1984-1988\end{array}$}} \\
\hline & \multirow[t]{2}{*}{ Hommes } & \multirow[t]{2}{*}{ Femmes } & \multirow[t]{2}{*}{ Total } & & & \\
\hline & & & & & $(\%)$ & (années) \\
\hline 11 - Lac St-Louis & 77.4 & 81.7 & 79,6 & 78,7 & 1,1 & 0,8 \\
\hline 13 - Plerrefonds & 72.9 & 78.1 & 75.6 & 75,8 & $-0,2$ & $-0,2$ \\
\hline 14 - Dollard-des-Ormeaux & 75.8 & 80.8 & 78.5 & 77,2 & 1.6 & 1.3 \\
\hline 15 - Vieux Lachine & 71,8 & 79,2 & 75,6 & 74,9 & 1.0 & 0,8 \\
\hline 21-Pointe St-Charles & 66.2 & 73,5 & 69,8 & 68,9 & 1,3 & 0,9 \\
\hline 22 - Verdun & 70.7 & 78.7 & 74.9 & 72,9 & 2.7 & 1,9 \\
\hline 24 - Ville-Émard-Côte St-Paul & 71.3 & 78.4 & 75.1 & $\mathbf{7 4 . 6}$ & 0.6 & 0.5 \\
\hline 26 - Lasalle & 73,0 & 79,5 & 76,5 & 75,2 & 1.6 & 1.2 \\
\hline 31 - Rivière-des-Prairies & $\mathbf{7 5 , 0}$ & 81,9 & 79,0 & 78,5 & 0.7 & 0,6 \\
\hline 32 - Octave-Roussin & 70,9 & 77.1 & 74,0 & 71,4 & 3,8 & 2.7 \\
\hline 33 - Mercier-Est & 69.8 & 78,4 & 73.9 & 73.0 & 1,3 & 1.0 \\
\hline 34 - Olivier-Guimond & 71,7 & 77.5 & 74,8 & 74,0 & 1.0 & 0.8 \\
\hline 35 - Hochelaga-Maisonneuve & 67,5 & 77,1 & 72,3 & 71,4 & 1,3 & 0,9 \\
\hline 36 - Rosemont & 71.1 & 79.3 & 75.5 & 74,6 & 1,3 & 1.0 \\
\hline 38 - Anjou & 73,3 & 81,0 & 77,6 & 77,3 & 0.5 & 0.4 \\
\hline 39 - St-Léonard & 75.2 & 81,4 & 78.5 & 77.2 & 1.6 & 1,3 \\
\hline 41 -Cóte-des-Neiges & 76,7 & 82,7 & 80,1 & 79,1 & 1,3 & 1,0 \\
\hline 42 - Snowdon & 74,0 & 80,7 & 77,5 & 78,0 & -0.7 & -0.5 \\
\hline 43 - Côte-St-Luc-Hampstead & 77.6 & 81.0 & 79,5 & 78,4 & 1,4 & 1,1 \\
\hline 44 - Outremont-Mont-Royal & 78.5 & $\mathbf{8 3 . 6}$ & 81.5 & 79.9 & 2.1 & 1,7 \\
\hline 51 - NDG-Montréal-Ouest & 74,3 & 80,5 & 77.7 & 76,1 & 2.1 & 1.6 \\
\hline 52 - Métro (Westmount) & 76,9 & 82,5 & 80,0 & 77,8 & 2,9 & 2,2 \\
\hline 53 - Métro (Centre Sud-Ouest) & 69.2 & 79.0 & 74.1 & 74,0 & 0,3 & 0,2 \\
\hline 54 - St-Louis du Parc & 69,2 & 77,4 & 73.1 & 70.9 & 3.2 & 2.3 \\
\hline 55 - St-Henri-Petite Bourgogne & 67,0 & 75,5 & 71,1 & 69,4 & 2,5 & 1.7 \\
\hline 61 - Montréal-Nord & 71,3 & 78,8 & 75,4 & $\mathbf{7 4 . 8}$ & 0.7 & 0,5 \\
\hline 63 - St-Michel & 72,6 & 79,3 & 76,2 & 75.9 & 0,5 & 0,4 \\
\hline 65 - Ahuntsic & 73,1 & 79,8 & 76,7 & 75,7 & 1,3 & 1.0 \\
\hline 66 - Bordeaux-Cartierville & $\mathbf{7 4 , 4}$ & 80,6 & 77,9 & 76,9 & 1,3 & 1,0 \\
\hline 68 - St-Laurent & 74.7 & 81,2 & 78.3 & 76.7 & 2,0 & 1.5 \\
\hline 71 - Centre-Sud & 66,2 & 75.7 & 70,6 & 68,5 & 3,0 & 2,0 \\
\hline 72 - Plateau Mont-Royal & 66.9 & 76,4 & 71,6 & 70,1 & 2,2 & 1,5 \\
\hline 74 - Parc Extension & 74,3 & 80,4 & 77.6 & 77,2 & 0,5 & 0,4 \\
\hline 75 - Centre-Ville & 68,5 & 77,2 & 72,3 & 68,6 & 5,4 & 3.7 \\
\hline 76 - Villeray & 71,7 & 79,7 & 76,0 & 75,3 & 0,9 & 0.7 \\
\hline 77 - Petite Patrie & 70.8 & 77.8 & 74.5 & 73.3 & 1,6 & 1,2 \\
\hline 81 - Des Mille-Îles & 74.5 & 79.5 & 77.2 & 75.6 & 2.1 & 1,6 \\
\hline 83 - Norman-Bethune & 73,8 & 79.4 & 76,8 & 75,5 & 1.7 & 1,3 \\
\hline 85 - Du Marigot & 73.0 & 80,8 & 77.1 & 75,7 & 1,8 & 1,4 \\
\hline 87 - Ste-Rose de Laval & 72.4 & 78.0 & 75.2 & 74,2 & 1,3 & 1.0 \\
\hline Région de Montréal & 72,5 & 79,5 & 76,2 & 75,0 & 1,5 & 1,2 \\
\hline
\end{tabular}

Sources: MSSS, fichier des naissances et des décès, 1979 à 1988; Choinière, 1990a; Wilkins, 1987 
De 1979-1983 à 1984-1988, la longévité moyenne de la population montréalaise est passée de 75,0 ans à 76,2 ans. La situation observée durant la période précédente, 1979-1983, est sensiblement la même que celle de la période 1984-1988. Les durées moyennes les plus faibles se retrouvent aussi dans la partie centre-sud de Montréal, particulièrement dans les CLSC Centre-Sud (68,5 ans), Centre-Ville (68,6 ans), Pointe SaintCharles (68,9 ans) et Saint-Henri-Petite Bourgogne (69,4 ans). L'espérance de vie à la naissance est supérieure à 79 ans dans Côte-des-Neiges et Outremont-Mont-Royal. L'écart entre les valeurs extrêmes est de 11,4 ans en 1979-1983; c'est un écart semblable à celui observé durant la dernière pérłode. Cinq territoires ont enregistré une hausse de l'espérance de vie égale ou supérieure à deux ans : Centre-Ville ( 3,7 ans), OctaveRoussin (2,7 ans), Saint-Louis du Parc (2,3 ans), Westmount (2,2 ans) et Centre-Sud (2,0 ans). Les populations de Pierrefonds et de Snowdon ont vu leur longévitê moyenne diminuer légèrement d'une période à l'autre.

Les disparités géographiques de l'espérance de vie n'ont pas diminué de 1979-1983 à 1984-1988. Guillemette (1983) montre de son côté, à partir d'un découpage de l'île de Montréal en douze zones socio-ẻconomiques, que l'écart en espérance de vie entre les zones extrêmes est passé de 8,9 ans en 1961 à 15,7 ans en 1976.

\section{LE TAUX DE MORTALITE INFANTILE}

Le taux de mortalité infantile a longtemps constitué un indicateur privilégiè des conditions d'hygiène et de santê régnant dans une population. Il permet de mettre en évidence des catégories de la population dont les conditions sont particulièrement défavorables (Péron et Strohmenger, $1985: 196$ ). Wilkins, Adams et Brancker (1989) montrent, pour les régions urbaines du Canada, qu'en termes relatifs le taux de mortalité infantile du quintile de revenu le plus bas est près de deux fois plus élevé que celui du quintile le plus haut, en 1986 comme en 1971. En termes absolus, cependant, l'écart entre les quintiles extrêmes a êté réduit de moitiê entre 1971 et 1986.

La plus grande part de l'aụgmentation de l'espérance de vie à la naissance observée au Canada entre 1931 et 1981 est due à la chute de la mortalité infantile. Cette contribution de la baisse de la mortalité infantile à l'espérance de vie diminue cependant de plus en plus (Péron et Strohmenger, 1985 : 119). 
Durant la période 1984-1988, la population montréalaise enregistre une moyenne de sept décès infantiles pour mille naissances vivantes. Les taux de mortalité infantile les plus faibles se retrouvent dans le West Island, dans le centre de l'île de Montréal et de Laval ainsi que dans Octave-Roussin (carte 2). Dans Westmount $(2,1$ p. 1000), Dollard-des-Ormeaux $(3,2$ p. 1000$)$, Côte-Saint-Luc-Hampstead $(3,8$ p. 1000) et Pierrefonds $(4,8 \mathrm{p} .1000)$, les taux de mortalité infantile sont inférieurs à $5 \mathrm{p}$. 1000 . Les niveaux les plus élevés sont observés dans les parties centre-sud et centre-est de Montréal, ainsi que dans Parc Extension et Mercier-Est. Deux territoires, SaintHenri-Petite Bourgogne (10,7 p. 1000) et Centre-Ville $(17,8$ p. 1000 ) présentent des taux dépassant 10 pour mille.

Le nombre de décès infantlles observés étant très petit, peu de territoires présentent un écart statistiquement significatif par rapport à l'ensemble de la région (tableau 5). Ce n'est que dans Centre-Ville que l'on retrouve, durant la période 19841988, une mortalité infantile significativement supérieure à la moyenne montréalaise.

À l'opposé, le taux enregistré dans Dollard-des-Ormeaux est le seul à être significativement plus faible que celui de la région. L'on ne sera pas surpris aussi de constater des variations importantes d'une période à l'autre. Ainsi, dans CentreVille, le taux est passé de 2,9 pour mille à 17,8 pour mille d'une période à l'autre, tandis que dans Westmount il a chuté de 10,3 pour mille à 2,1 pour mille. Cependant, exception faite de l'ensemble de la région, les différences observées dans les taux de mortalité infantile entre les deux périodes ne sont significatives pour aucun territoire. Le portrait général est le même pour les deux périodes. La mortalité infantile est faible dans l'ouest et le centre de l'île et élevêe dans les parties centre-sud et centreest. À l'échelle de l'ensemble du territoire montréalais, la mortalité infantile présente une baisse de près de $20 \%$ de 1979 1983 à 1984-1988, passant de 8,6 pour mille à 7,0 pour mille.

\section{DISCUSSION}

Les deux mesures retenues, l'espérance de vie à la naissance et le taux de mortalité infantile, révèlent des disparités géographiques importantes de la mortalitê dans la région de Montrêal. Ces disparités ne semblent pas avoir diminué d'une période à l'autre. Les territoires situés dans la partie centre-sud de l'île de Montréal présentent une mortalité nettement supé- 
웅 赵乐

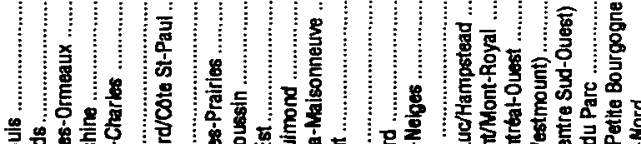

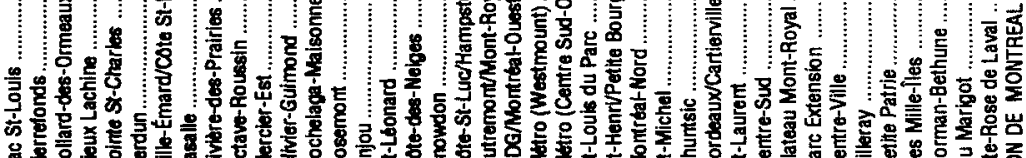

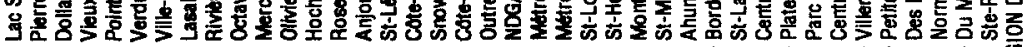

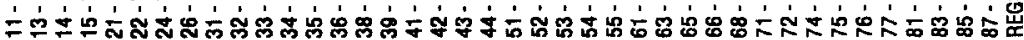

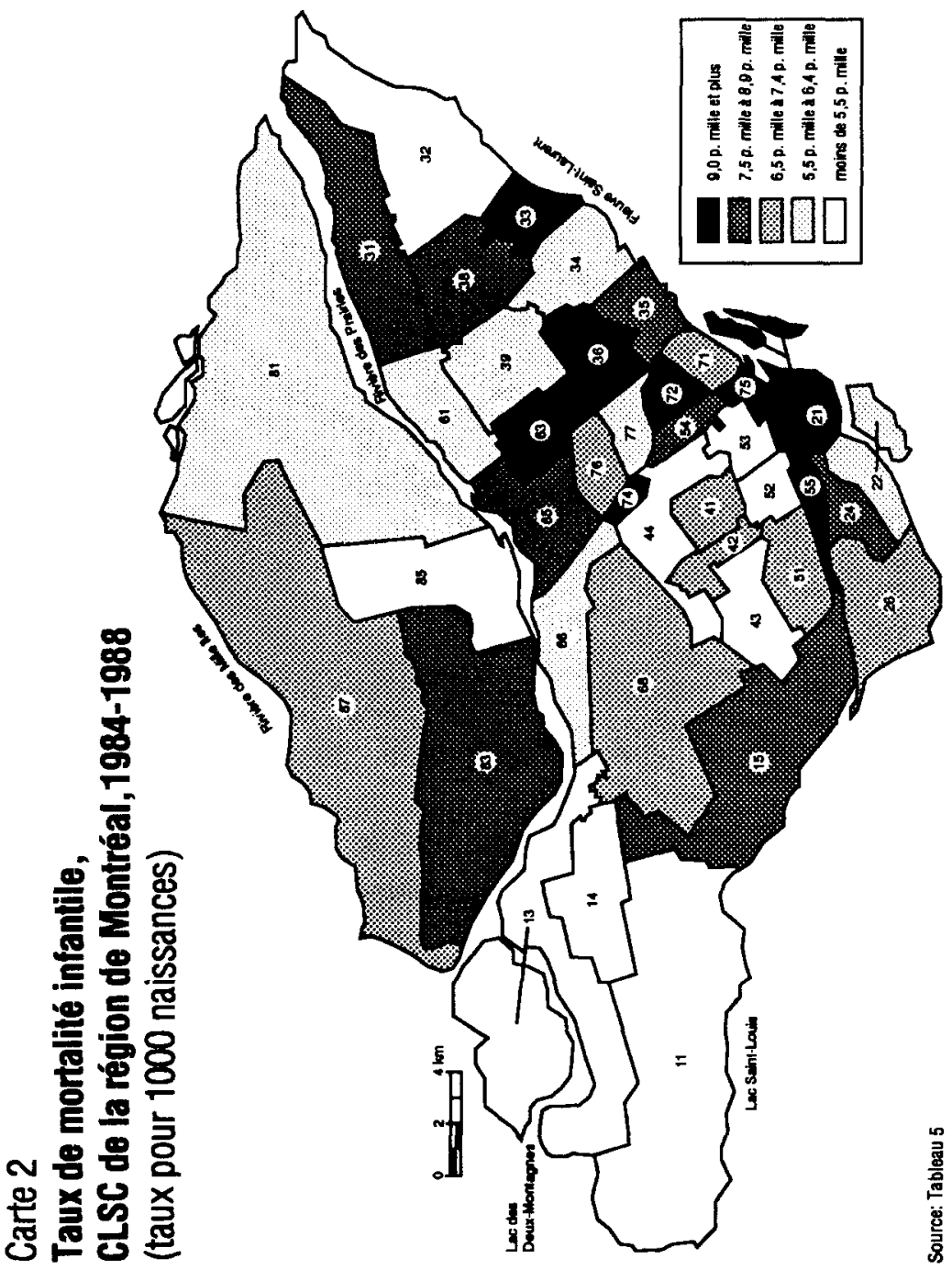


TABLEAU 5

Taux de mortalité infantile, CLSC de la région de Montréal, 1979-1983 et 1984-1988 (taux pour 1000 naissances)

\begin{tabular}{|c|c|c|c|c|c|c|}
\hline \multirow[b]{3}{*}{ Territoires } & \multicolumn{3}{|c|}{$1984-1988$} & \multicolumn{3}{|c|}{$1979-1983$} \\
\hline & \multicolumn{3}{|c|}{ Intervalle de confiance ${ }^{\mathbf{a}}$} & \multicolumn{3}{|c|}{ Intervalle de confiance ${ }^{a}$} \\
\hline & Taux & $\begin{array}{l}\text { Limite } \\
\text { infér. }\end{array}$ & $\begin{array}{l}\text { Limite } \\
\text { supér. }\end{array}$ & Taux & $\begin{array}{c}\text { Limite } \\
\text { infêr. }\end{array}$ & $\begin{array}{l}\text { Limite } \\
\text { supér. }\end{array}$ \\
\hline 11 - Lac St-Louis & 5,0 & 3.2 & 7.6 & 7,0 & 4,8 & 10.1 \\
\hline 13 - Pierrefonds & 4,8 & 3.1 & 7.4 & 6,9 & 4.7 & 10,0 \\
\hline 14 - Dollard-des-Ormeaux & 3,2 & 1,6 & 6.1 & 6,9 & 4,5 & 10,7 \\
\hline 15 - Vieux Lachine & 8,1 & 5,5 & 11,8 & 8,6 & 5,9 & 12,3 \\
\hline 21 - Pointe St-Charles & 9.2 & 4,5 & 18,0 & 13,9 & 7,8 & 24,4 \\
\hline 22 - Verdun & 6,3 & 4.1 & 9,6 & 9.1 & 6,3 & 13,1 \\
\hline 24 - Ville-Émard-Cōte St-Paul & 8,3 & 4,8 & 14,0 & 10,0 & 6,2 & 15,9 \\
\hline 26 - Lasalle & 6,7 & 4.7 & 9,4 & 7.0 & 5,0 & 9,8 \\
\hline 31 - Rivière-des-Prairies & 8,0 & 5,1 & 12,4 & 8,7 & 4,9 & 15,3 \\
\hline 32 - Octave-Roussin & 5,1 & 3,1 & 8,3 & 11.7 & 8,2 & 16,7 \\
\hline 33 - Mercier-Est & 9.2 & 6,1 & 13,8 & 11.5 & 8,0 & 16.5 \\
\hline 34 - Olivier-Guimond & 6,0 & $\mathbf{3 , 3}$ & 10,5 & 7.2 & 4.4 & 11,7 \\
\hline 35 - Hochelaga-Maisonneuve & 8,0 & 5,3 & 12,1 & 11.5 & 8,1 & 16,3 \\
\hline 36 - Rosemont & 9,0 & 6,4 & 12.5 & 8,2 & 5.8 & 11.6 \\
\hline 38 - Anjou & 8,7 & 5,3 & 14,2 & 6,6 & 3.9 & 11,2 \\
\hline 39 - St-Léonard & 5,9 & 4,1 & 8,5 & 7.7 & 5,8 & 10,4 \\
\hline 41 - Côte-des-Neiges & 6.6 & 4,1 & 10,3 & 6,3 & 3,9 & 10.2 \\
\hline 42 - Snowdon & 6.7 & 3.9 & 11,3 & 7.9 & 4.6 & 13.3 \\
\hline 43 - Còte-St-Luc-Hampstead & $\mathbf{3 , 8}$ & 1,8 & 7,8 & 7,4 & 4,2 & 12,8 \\
\hline 44 - Outremont-Mont-Royal & 5,2 & 2,5 & 10,2 & 7,3 & 4.2 & 12,6 \\
\hline 51 - NDG-Montréal-Ouest & 7,4 & 5.1 & 10,6 & 6.8 & 4.6 & 10.0 \\
\hline 52 - Métro (Westmount) & 2,1 & 0.4 & 8,6 & 10.3 & 5.0 & 20.1 \\
\hline 53 - Métro (Centre Sud-Ouest) & 5,5 & 2.6 & 11,2 & 13,4 & 8,3 & 21,2 \\
\hline 54 - St-Louis du Parc & 8,4 & 5,6 & 12.4 & 11,9 & 8,7 & 16,3 \\
\hline 55 - St-Henri-Petite Bourgogne & 10.7 & 6.2 & 17.9 & 9.2 & 5,0 & 16.4 \\
\hline 61 - Montréal-Nord & 6,3 & 4,5 & 8,9 & 7,0 & 5,2 & 9,4 \\
\hline 63 - St-Michel & 9.4 & 6.6 & 13,1 & 8,5 & 6,0 & 11,9 \\
\hline 65 - Ahuntsic & 8,3 & 5,9 & 11.6 & 8.8 & 6.2 & 12.3 \\
\hline 66 - Bordeaux-Cartierville & 5,9 & 3,5 & 9,8 & 9,1 & 5,7 & 14,2 \\
\hline 68 - St-Laurent & 6.9 & 4,6 & 10,5 & 8,5 & 5,8 & 12,2 \\
\hline 71 - Centre-Sud & 7.3 & 4,1 & 12.8 & 13,2 & 8.9 & 19,4 \\
\hline 72 - Plateau Mont-Royal & 9.5 & 6.3 & 14.1 & 14,2 & 10,4 & 19.4 \\
\hline 74 - Parc Extension & 9,6 & 6,2 & 14.7 & 11,4 & 7,7 & 16,6 \\
\hline 75 - Centre-Ville & 17,8 & 7.8 & 38,0 & 2,9 & 0,2 & 18.5 \\
\hline 76 - Villeray & 7,1 & 4,8 & 10,4 & 9,2 & 6,5 & 13,0 \\
\hline 77 - Petite Patrie & 6.2 & 3,9 & $\mathbf{9 . 9}$ & 8,0 & 5,2 & 12,0 \\
\hline 81 - Des Mille-îles & 6.1 & 3.5 & 10.3 & 8,3 & 5.2 & 13,0 \\
\hline 83 - Norman-Bethune & 7,5 & 5.3 & 10,4 & 7.6 & 5.4 & 10.7 \\
\hline 85 - Du Marigot & 5,0 & $\mathbf{3 , 3}$ & 7,4 & 8,2 & 6,0 & 11,1 \\
\hline 87 - Ste-Rose de Laval & 6,5 & 4,7 & 9,0 & 7,7 & 5,6 & 10,5 \\
\hline Région de Montréal & 7.0 & 6.6 & 7.5 & 8,6 & 8,1 & 9,1 \\
\hline
\end{tabular}

Source : MSSS, fichier des naissances et des décès, 1979 à 1988.

a Intervalles de confiance à $95 \%$ calculés selon la méthode décrite dans Fleiss, 1981 (p. 13-15). 
rieure à la moyenne montréalaise, tandis qu'au centre et à l'extrémité ouest de l'île de Montréal la mortalité se situe à un niveau beaucoup plus bas.

L'on peut se demander si ces disparités géographiques de la mortalitê ne correspondent pas à des disparitês d'ordre social, culturel ou économique. L'étude de la mortalité différentielle selon le statut socio-économique a fait l'objet de nombreux travaux, dont quelques-uns ont êté mentionnés plus haut. D'autres études, moins nombreuses, se sont penchées sur les différences de mortalité à partir de caractéristiques culturelles (groupes linguistiques, groupes ethniques, population immigrante) ${ }^{1}$.

La carte 3 décrit la proportion de personnes vivant sous le seuil de pauvreté pour chacun des quarante territoires. Dans l'ensemble de la région, une personne sur quatre serait pauvre. Les proportions les plus élevées (en noir) se retrouvent dans la partie centre-sud de l'île de Montréal. Dans les CLSC Pointe Saint-Charles (52\%), Centre-Ville (51\%), Parc Extension (49\%), Centre-Sud (48\%), Saint-Henri-Petite Bourgogne (46\%), Saint-Louis du Parc (41\%) et Hochelaga-Maisonneuve (40\%), $40 \%$ et plus de la population vit sous le seuil de pauvreté. Les plus faibles proportions de personnes pauvres len blanc) se situent à l'extrémité ouest de l'île de Montréal et à Laval, ainsi que dans Westmount et Outremont-Mont-Royal.

Les disparités géographiques de la mortalité dans la région de Montréal semblent s'apparenter à celles de la pauvreté. Dans la partie centre-sud, l'on retrouve à la fois les niveaux de mortalité les plus élevés et les plus fortes proportions de personnes pauvres.

Le graphique 1 présente le diagramme de dispersion obtenu à partir de la régression entre l'espérance de vie à la naissance et la proportion de personnes pauvres pour les territoires étudiés. Le diagramme et la droite de rêgression illustrent bien le sens de la relation entre l'espérance de vie et la pauvreté. L'espérance de vie diminue à mesure que la proportion de personnes pauvres augmente. Le coefficient de détermination, quil représente le pourcentage de la variation de l'espérance de vie expliquée par la droite de régression, est de 0,47 . Ainsi, un peu moins de la moitié des variations géographiques de l'espérance de vie serait reliée aux variations du taux de pauvretê.

1 Voir Wilkins, Adams et Brancker (1989) pour une revue récente de la littérature sur ces sujets. 


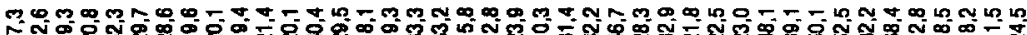
П

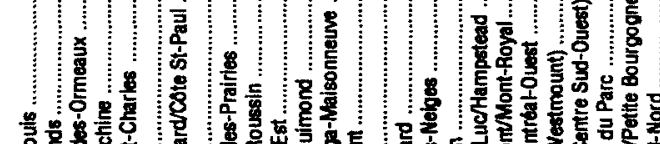

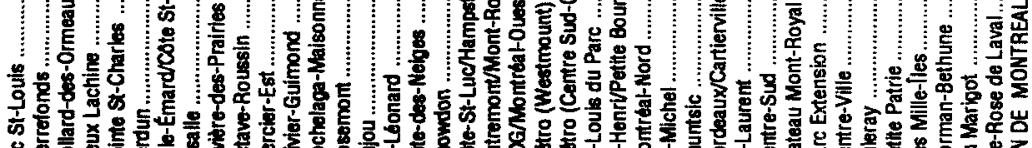

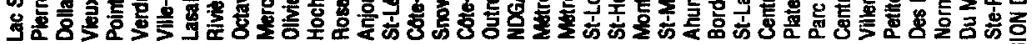

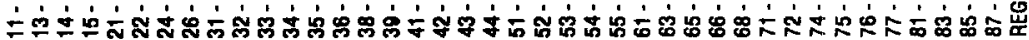

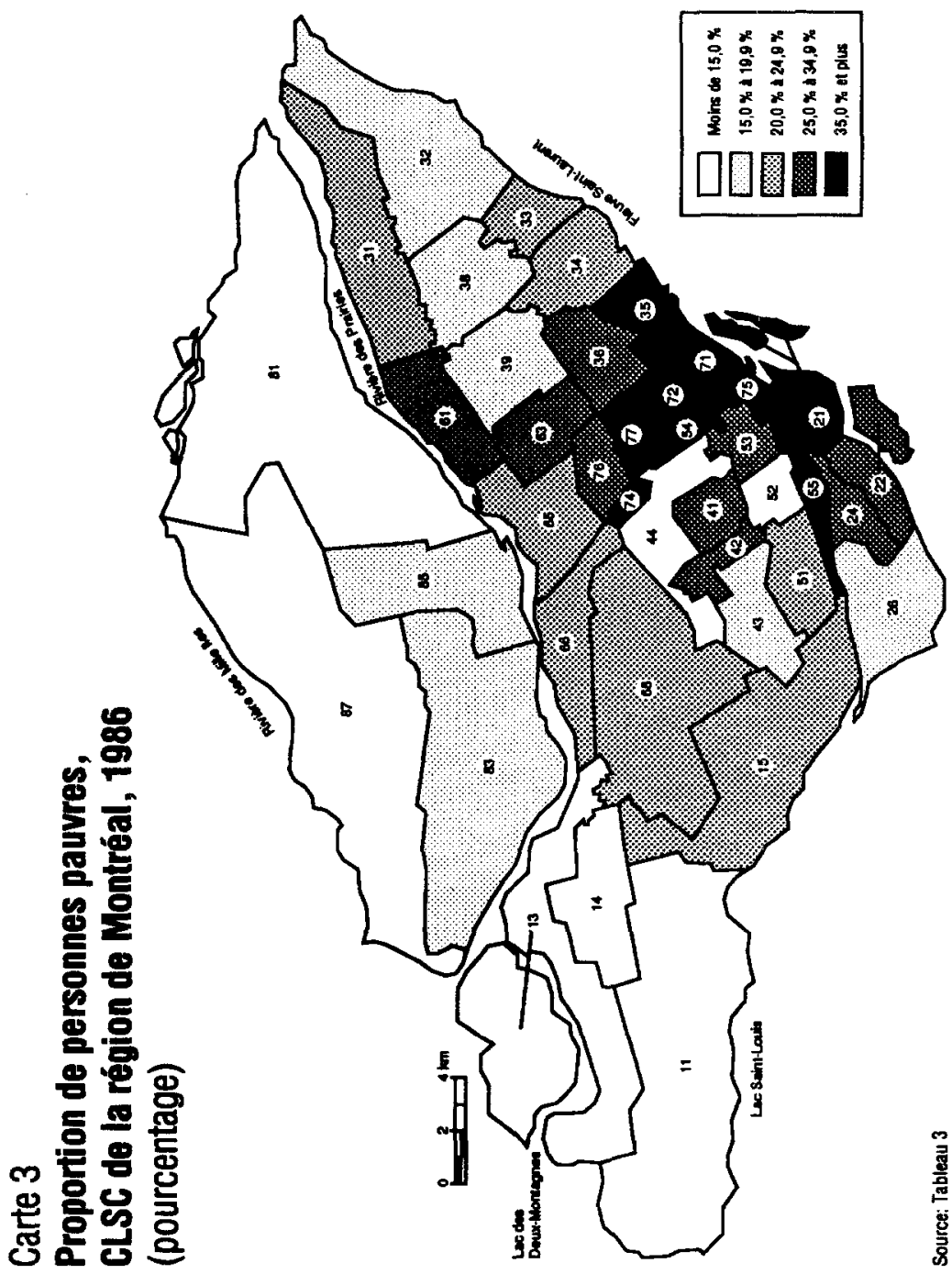




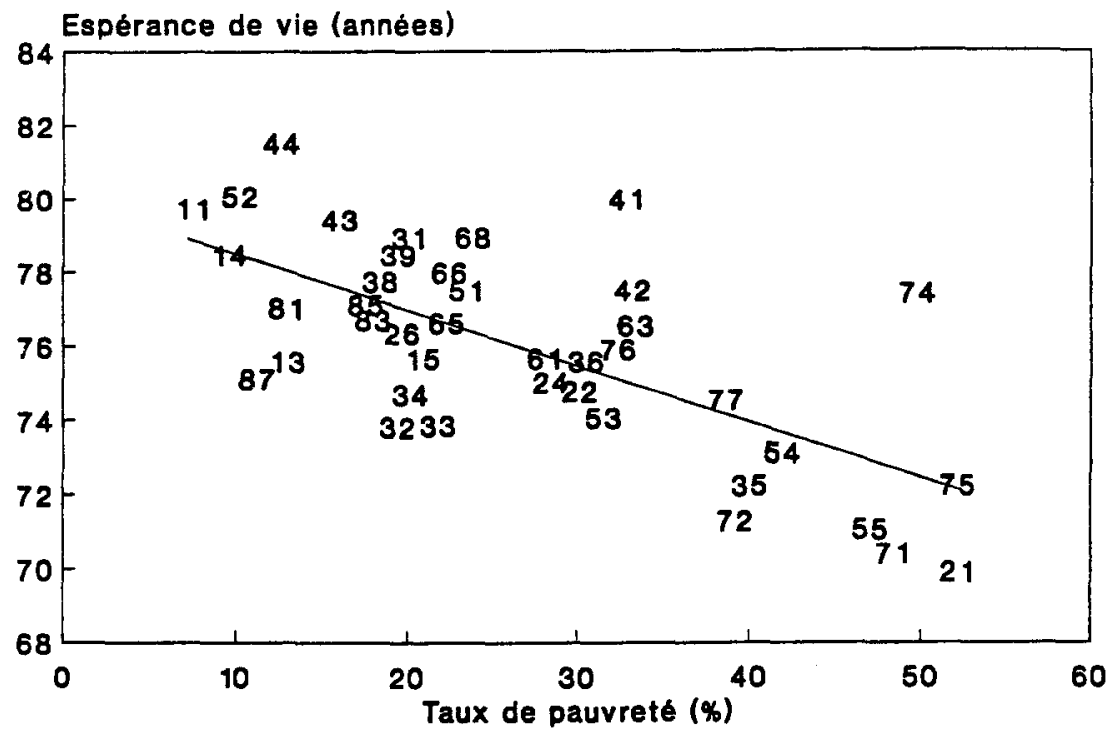

Sounces : tableau 3 et carte 3.

11 Lac St-Louis
13 Pierrefonds
14 Dollard-des-
Ormeaux
15 Vieux Lachine
21 Pointe St-Charles
22 Verdun
24 Ville-Êmard-Côte-
St-Paul
26 LaSalle
31 Rivière-des-Prairies
32 Octave-Roussin
33 Mercier-Est
34 Olivier-Guimond
35 Hochelage-
Maisonneuve

36 Rosemont
38 Anjou
39 St-Léonard
41 Côte-des-Neiges
42 Snowdon
43 Côte-St-Luc-
Hampstead
44 Outremont-Mont-
Royal
51 NDG-Montreaal-Ouest
52 Métro (Westmount)
53 Métro (Centre sud-
ouest)
54 St-Louis du Parc
55 St-Henri-Petite
Bourgogne

61 Montréal-Nord

63 St-Michel

65 Ahuntsic

66 BordeauxCartierville

68 St-Laurent

71 Centre-Sud

72 Plateau Mont-Royal

74 Parc Extension

75 Centre-Ville

76 Villeray

77 Petite Patrie

81 Des Mille-Î̀les

83 Norman-Bethune

$85 \mathrm{Du}$ Marigot

87 Ste-Rose de Laval

\section{Graphique 1 - ESPERANCE DE VIE A LA NAISSANCE} SELON LE TAUX DE PAUVRETE

Certains territoires s'écartent sensiblement de la droite. Par exemple, Parc Extension (numéro 74 sur le graphique) présente une espérance de vie élevée $(77,6$ ans), bien que la moitié de sa population se situe sous le seuil de pauvreté. À l'inverse, Sainte-Rose de Laval (numéro 87) enregistre une espérance de vie plutôt basse $(75,2$ ans) alors que l'on retrouve un faible pourcentage de pauvres $(11,5 \%)$ sur ce territoire.

Le tableau 6 reproduit les coefficients de corrélation et de détermination simples obtenus entre les deux indicateurs de 
TABLEAU 6

Coefficients de corrélation ( $r$ ) et de détermination ( $r$ carré) simples

\begin{tabular}{lrrrr}
\hline & \multicolumn{2}{c}{$\begin{array}{c}\text { Espérance de vie } \\
\text { Vara la naissance }\end{array}$} & \multicolumn{2}{c}{$\begin{array}{c}\text { Taux de mortalité } \\
\text { infantile }\end{array}$} \\
indépendantes & r & r carré & \multicolumn{1}{c}{ r } & r carré \\
\hline \% de pauvres & $-0,68$ & 0,47 & 0,68 & 0,46 \\
\% d'immigrants & 0,50 & 0,25 & $-0,02$ & 0,00 \\
\% de personnes inoccupées & $-0,64$ & 0,41 & 0,55 & 0,31 \\
\% de personnes ayant moins & & & & \\
de 9 ans de scolarité & $-0,61$ & 0,37 & 0,58 & 0,34 \\
\% de familles monoparentales & $-0,71$ & 0,51 & 0,57 & 0,32 \\
\% de naissances de faible poids & $-0,71$ & 0,51 & 0,31 & 0,10 \\
\% de personnes ayant une & & & & \\
profession dans les secteurs & $-0,36$ & 0,13 & 0,29 & 0,08 \\
primaire ou secondaire & & & & \\
Rapport de dépendance & 0,38 & 0,14 & $-0,12$ & 0,01 \\
\hline
\end{tabular}

Sources : tableaux 3, 4 et 5 .

mortalité et chacune des huit variables indépendantes retenues dans cette étude. Un signe négatif précédant le coefficient de corrélation indique une relation opposée entre la variable indépendante et la variable dépendante.

Les corrélations observées diffèrent entre l'espérance de vie et la mortalité infantlle. Exception faite du pourcentage de personnes pauvres, l'espérance de vie prêsente des associations plus fortes que la mortalité infantile avec les variables étudiées. Des coefficients de détermination égaux ou supérieurs à $25 \%$ sont obtenus entre l'espérance de vie et les proportions de familles monoparentales $(0,51)$, de naissances de faible poids $(0,51)$, de pauvres $(0,47)$, de personnes ayant moins de 9 ans de scolarité $(0,37)$ et d'immigrants $(0,25)$. Le taux de mortalité infantile enregistre des coefficients de détermination supérieurs à $25 \%$ avec les proportions de pauvres $(0,46)$, de personnes ayant moins de 9 ans de scolarité $(0,34)$, de familles monoparentales $(0,32)$ et de personnes inoccupées $(0,31)$.

L'on peut passer de la régression simple à la régression multiple en utilisant plus d'une variable indépendante à la fois pour expliquer les variations géographiques de la mortalité. Le tableau 7 présente, pour l'espérance de vie à la naissance, les résultats obtenus selon le nombre de variables indépendantes retenues. Les variables ont êté ajoutées une à une au pourcen- 
TABLEAU 7

Régression multiple de l'espérance de vie à la naissance, 1984-1988 a

$\begin{array}{llllllllll}\begin{array}{l}\text { Variables } \\ \text { indépendantes }\end{array} & \text { (1) } & \text { (2) } & \text { (3) } & \text { (4) } & \text { (5) } & \text { (6) } & \text { (7) } & \text { (8) Bèta }\end{array}$

\begin{tabular}{|c|c|c|c|c|c|c|c|c|c|c|}
\hline (1) \% de pauvres & & $\begin{array}{ll}\text { B } & -0,15 \\
\text { t } & (-5,81)(-\end{array}$ & $\begin{array}{l}-0,17 \\
-10,63)\end{array}$ & $\begin{array}{l}-0,23 \\
(-7.28)\end{array}$ & $\begin{array}{c}-0,24 \\
(-7,04)\end{array}$ & $\begin{array}{c}-0,27 \\
(-4,68)\end{array}$ & $\begin{array}{c}-0,27 \\
(-4,61)\end{array}$ & $\begin{array}{c}-0,27 \\
(-4,53)\end{array}$ & $\begin{array}{c}-0,27 \\
(-4,16)\end{array}$ & $-1,20$ \\
\hline $\begin{array}{l}\text { (2) \% d'immi- } \\
\text { grants }\end{array}$ & & $\begin{array}{l}B \\
t\end{array}$ & $\begin{array}{c}0,12 \\
(8,32)\end{array}$ & $\begin{array}{c}0,14 \\
(8,95)\end{array}$ & $\begin{array}{c}0.14 \\
(8.71)\end{array}$ & $\begin{array}{r}0.15 \\
(7,25)\end{array}$ & $\begin{array}{c}0.14 \\
(6,37)\end{array}$ & $\begin{array}{c}0.14 \\
(6,26)\end{array}$ & $\begin{array}{c}0,14 \\
(5,40)\end{array}$ & 0.70 \\
\hline $\begin{array}{l}\text { (3) \% de personnes } \\
\text { inoccupées }\end{array}$ & & $\begin{array}{l}B \\
t\end{array}$ & & $\begin{array}{c}0.13 \\
(2.29)\end{array}$ & $\begin{array}{c}0,12 \\
(1,93)\end{array}$ & $\begin{array}{c}0.11 \\
(1,69)\end{array}$ & $\begin{array}{c}0.12 \\
(1,70)\end{array}$ & $\begin{array}{c}0,12 \\
(1,54)\end{array}$ & $\begin{array}{c}0,12 \\
(0,86)\end{array}$ & 0.29 \\
\hline $\begin{array}{l}\text { (4) \% de personnes } \\
\text { ayant moins de } \\
9 \text { ans de scolanté }\end{array}$ & & $\begin{array}{l}B \\
t\end{array}$ & & & $\begin{array}{c}0.02 \\
(0.51)\end{array}$ & $\begin{array}{c}0.04 \\
(0.80)\end{array}$ & $\begin{array}{c}0.04 \\
(0.85)\end{array}$ & $\begin{array}{c}0.03 \\
(0,30)\end{array}$ & $\begin{array}{c}0.03 \\
(0,29)\end{array}$ & 0,10 \\
\hline $\begin{array}{l}\text { (5) } \% \text { de familles } \\
\text { monoparentales }\end{array}$ & & $\begin{array}{l}B \\
t\end{array}$ & & & & $\begin{array}{c}0.03 \\
(0.65)\end{array}$ & $\begin{array}{c}0.03 \\
(0.68)\end{array}$ & $\begin{array}{c}0.04 \\
(0.64)\end{array}$ & $\begin{array}{c}0.04 \\
(0,58)\end{array}$ & 0.15 \\
\hline $\begin{array}{l}\text { (6) \% de nais- } \\
\text { sances de } \\
\text { faible poids }\end{array}$ & $\begin{array}{l}\mathrm{E} \\
\mathrm{t}\end{array}$ & $\begin{array}{l}B \\
t\end{array}$ & & & & & $\begin{array}{c}-0,10 \\
(-0,33)\end{array}$ & $\begin{array}{c}-0,13 \\
(-0,36)\end{array}$ & $\begin{array}{c}-0.13 \\
(-0,35)\end{array}$ & $-0,06$ \\
\hline $\begin{array}{l}\text { ( } 7 \text { ) \% de personnes } \\
\text { ayant une pro- } \\
\text { fession dans les } \\
\text { secteurs primaire } \\
\text { ou secondaire }\end{array}$ & & $\begin{array}{l}B \\
t\end{array}$ & & & & & & $\begin{array}{c}0.01 \\
(0,16)\end{array}$ & $\begin{array}{c}0,01 \\
(0,16)\end{array}$ & 0.04 \\
\hline $\begin{array}{l}\text { (8) Rapport de } \\
\text { dépendance }\end{array}$ & & B & & & & & & & $\begin{array}{c}0,00 \\
(0,01)\end{array}$ & 0,00 \\
\hline Constante & $\begin{array}{l}\text { B } \\
t\end{array}$ & $\begin{array}{c}80.02 \\
(104.38)(\end{array}$ & $\begin{array}{l}77,84 \\
147,43)\end{array}$ & $\begin{array}{l}73.26 \\
(35,48)\end{array}$ & $\begin{array}{l}73,48 \\
\{34,49\}\end{array}$ & $\begin{array}{l}73,20 \\
(33,41)\end{array}$ & $\begin{array}{l}73.58 \\
(29,43)\end{array}$ & $\begin{array}{l}73,33 \\
(24,94)\end{array}$ & $\begin{array}{l}73,34 \\
(24,43\}\end{array}$ & \\
\hline carré & & 0,47 & 0.82 & 0.84 & 0.84 & 0.84 & 0,84 & 0.84 & 0.84 & \\
\hline
\end{tabular}

Sources : tableaux 3, 4 et 5 .

a $\quad B$ = coefficient de régression;

$t=\frac{\text { coefficient de régression }}{\text { erreur type du coefficient }}$

Bèta $=$ coefficient de régression standardisé.

tage de personnes pauvres de façon à obtenir, chaque fois qu'une nouvelle variable était introduite, le coefficient de détermination maximum.

En plus du coefficient de détermination ( $r$ carré), l'on retrouve au tableau 7 le coefficient de régression "B*, le ratio "t" et le coefficient de régression standardisé "Bêta". "B" estime, pour une variable indépendante donnée, l'effet de ses variations sur les valeurs de la variable dépendante lorsque les autres variables indépendantes sont gardées constantes. On 
obtient le ratio "t" en divisant le coefficient de régression par l'erreur type. Ce ratio doit être supérieur à 2 pour que le coefficient de régression soit significatif à $95 \%$. Le coefficient "Bêta" mesure l'effet des variations de chaque variable indëpendante sur les valeurs de la variable dépendante en unités d'écart type, ce qui permet de comparer entre elles des variables ayant des unités de mesure différentes ${ }^{2}$.

Les huit variables indépendantes utilisées conjointement expliquent $84 \%$ des variations géographiques de l'espérance de vie. En ne retenant que trois variables, la proportion de personnes pauvres, le pourcentage d'immigrants et le taux d'inoccupation, l'on aurait obtenu le même résultat. À elles seules, les deux premières variables expliquent $82 \%$ des variations de l'espérance de vie. La proportion de personnes pauvres et le pourcentage d'immigrants sont d'ailleurs les deux facteurs qui entraînent les variations les plus importantes de l'espérance de vie ("Bêta" $=-1,20$ et 0,70 ).

Les proportions de familles monoparentales et de naissances de faible poids enregistrent les corrélations simples les plus élevées avec l'espérance de vie. Cependant, lorsque l'on isole chaque variable en contrôlant toutes les autres variables, l'impact respectif de ces deux variables sur l'espérance de vie devient faible. Le pourcentage de pauvres et la proportion d'immigrants représentent alors les facteurs les plus importants dans les variations géographiques de l'espérance de vie.

Une augmentation d'un point du pourcentage de pauvres se traduit par une diminution de 0,15 an en espérance de vie et de 0,27 an quand tous les autres facteurs sont contrôlés. Une hausse d'un pour cent de la proportion d'immigrants entraîne une augmentation de 0,12 an en espérance de vie lorsque le pourcentage de personnes pauvres est contrôlé, et de 0,14 an lorsque toutes les variables le sont.

Les coefficients de régression "B* obtenus pour les proportions de pauvres et d'immigrants sont tous significatifs à $95 \%$, peu importe le nombre de variables retenues dans l'équation, tandis que pour les autres variables, à une exception près, les coefficients ne sont pas significatifs (

2 Pour une description détaillée des méthodes utilisées dans l'analyse de régression, le lecteur pourra consulter Berry et Feldman, 1990; Kleinbaum. Kupper et Muller, 1987; Lewis-Beck, 1990; Schroeder, Sjoquist et Stephan, 1989. 
Le sens de la relation entre l'espérance de vie et les proportions de personnes ayant moins de 9 ans de scolarité, de familles monoparentales et de personnes exerçant une profession dans les secteurs primaire ou secondaire change lorsque l'on contrôle pour la pauvreté et l'immigration. Au tableau 6, l'on observe une corrélation négative entre ces variables et l'espêrance de vie, tandis qu'au tableau 7 les coefficients de régression " $B$ " montrent une relation positive entre ces variables indépendantes et l'espérance de vie.

Le mème exercice peut être fait pour le taux de mortalité infantile. Les résultats sont présentés au tableau 8 . Les huit variables indépendantes retenues dans le modèle expliquent $63 \%$ des variations géographiques de la mortalité infantile. Ce résultat est donc beaucoup plus faible que celui obtenu pour l'espérance de vie (84\%). Pour l'espérance de vie, l'utilisation de plus de trois variables ne permet pas d'augmenter le coefficient de détermination, tandis que pour la mortalité infantile, ce n'est qu'à partir de sept variables que le r carré cesse d'augmenter.

La proportion de pauvres et le taux d'inoccupation sont les deux facteurs qui occasionnent les plus grandes variations du taux de mortalité infantile. Cependant, les coefficients de régression observés pour le taux d'inoccupation ne sont pas significatifs.

Une augmentation d'un pour cent de la proportion de pauvres provoque une hausse de 0,14 pour mille du taux de mortalité infantile et de 0,22 pour mille lorsque toutes les autres variables sont contrôlées. En plus de la proportion de pauvres, deux autres variables (la proportion de nalssances de faible poids et la proportion d'immigrants) présentent des coefficients de régression " $B$ " significatifs. Bien que la proportion d'immigrants enregistre une corrélation simple presque nulle avec la mortalité infantile, celle-ci a un impact significatif sur les variations de la mortalité infantile lorsque les autres variables sont gardées constantes.

Pour quatre variables indépendantes (naissances de faible poids, scolaritê, inoccupation et rapport de dépendance), le sens de la relation avec la mortalité infantile change lorsque l'on passe de la régression simple à la régression multiple. Ainsi, lorsque la proportion de personnes pauvres reste constante, une augmentation d'un pour cent de la proportion de naissances de faible poids fait baisser le taux de mortalité infantile de 0,40 pour mille. Ce résultat semble surprenant de prime 
TABLEAU 8

Régression multíple du taux de mortalité infantile, 1984-1988

\begin{tabular}{|c|c|c|c|c|c|c|c|c|c|c|}
\hline $\begin{array}{l}\text { Variables } \\
\text { indépendantes }\end{array}$ & & (1) & (2) & (3) & (4) & (5) & (6) & (7) & (8) & Bêta \\
\hline (1) \% de pauvres & $\begin{array}{l}\mathrm{B} \\
\mathrm{t}\end{array}$ & $\begin{array}{c}0.14 \\
(5.70)\end{array}$ & $\begin{array}{c}0.16 \\
(5.22)\end{array}$ & $\begin{array}{c}0.21 \\
(5.91)\end{array}$ & $\begin{array}{r}0,17 \\
(4,35)\end{array}$ & $\begin{array}{c}0,20 \\
(3.87)\end{array}$ & $\begin{array}{c}0,27 \\
(3.66)\end{array}$ & $\begin{array}{c}0,22 \\
(2,50)\end{array}$ & $\begin{array}{c}0,22 \\
(2,45)\end{array}$ & 1.09 \\
\hline $\begin{array}{l}\text { (2) } \% \text { de nais- } \\
\text { sances de faible } \\
\text { poids }\end{array}$ & $\begin{array}{l}\mathrm{B} \\
\mathrm{t}\end{array}$ & & $\begin{array}{c}-0,40 \\
(-1,20)\end{array}$ & $\begin{array}{r}-1,06 \\
(-2,54)\end{array}$ & $\begin{array}{c}-1,32 \\
(-3,03)\end{array}$ & $\begin{array}{c}-1,24 \\
(-2,79)\end{array}$ & $\begin{array}{r}-1,15 \\
(-2,59)\end{array}$ & $\begin{array}{c}-1,19 \\
(-2,68)\end{array}$ & $\begin{array}{c}-1,29 \\
(-2.42)\end{array}$ & $-0,60$ \\
\hline (3) \% d'immigrants & $\begin{array}{l}\mathrm{B} \\
\mathrm{t}\end{array}$ & & & $\begin{array}{r}-0,07 \\
(-2,37)\end{array}$ & $\begin{array}{c}-0,06 \\
(-2.29)\end{array}$ & $\begin{array}{c}-0,06 \\
(-2,34)\end{array}$ & $\begin{array}{r}-0.10 \\
(-2.71)\end{array}$ & $\begin{array}{r}-0,09 \\
(-2.42)\end{array}$ & $\begin{array}{c}-0,09 \\
(-2,38)\end{array}$ & $-0,47$ \\
\hline $\begin{array}{l}\text { (4) } \% \text { de personnes } \\
\text { ayant moins de } \\
9 \text { ans de scolarité }\end{array}$ & $\begin{array}{l}B \\
t\end{array}$ & & & & $\begin{array}{c}0,08 \\
(1.70)\end{array}$ & $\begin{array}{c}0,09 \\
(1,83)\end{array}$ & $\begin{array}{r}0.13 \\
(2.28)\end{array}$ & $\begin{array}{c}0.19 \\
(2,39)\end{array}$ & $\begin{array}{c}0.16 \\
(1.19)\end{array}$ & 0.60 \\
\hline $\begin{array}{l}\text { (5) \% de personnes } \\
\text { tnoccupées }\end{array}$ & $\begin{array}{l}B \\
t\end{array}$ & & & & & $\begin{array}{c}-0,07 \\
(-0.74)\end{array}$ & $\begin{array}{r}-0,27 \\
(-1.57)\end{array}$ & $\begin{array}{l}-0,36 \\
(-1,90)\end{array}$ & $\begin{array}{l}-0,35 \\
(1.80)\end{array}$ & $-0,94$ \\
\hline $\begin{array}{l}\text { (6) Rapport de } \\
\text { dépendance }\end{array}$ & $\begin{array}{l}\mathrm{B} \\
\mathrm{t}\end{array}$ & & & & & & $\begin{array}{c}0,12 \\
(1,39)\end{array}$ & $\begin{array}{c}0,16 \\
(1,70)\end{array}$ & $\begin{array}{c}0.17 \\
(1.71)\end{array}$ & 0,40 \\
\hline $\begin{array}{l}\text { (7) \% de familles } \\
\text { monoparentales }\end{array}$ & $\begin{array}{l}B \\
t\end{array}$ & & & & & & & $\begin{array}{r}0,09 \\
(1,09)\end{array}$ & $\begin{array}{c}0.11 \\
(1,08)\end{array}$ & 0.43 \\
\hline $\begin{array}{l}\text { (8) \% de personnes } \\
\text { ayant une pro- } \\
\text { fession dans les } \\
\text { secteurs primaire } \\
\text { ou secondaire }\end{array}$ & $\begin{array}{l}\mathrm{B} \\
\mathrm{t}\end{array}$ & & & & & & & & $\begin{array}{c}0,03 \\
(0,35)\end{array}$ & 0,14 \\
\hline Constante & $\begin{array}{l}\mathrm{B} \\
\mathrm{t}\end{array}$ & $\begin{array}{r}3,52 \\
(4,98)\end{array}$ & $\begin{array}{c}5,49 \\
(3,09)\end{array}$ & $\begin{array}{r}9,98 \\
(3,95)\end{array}$ & $\begin{array}{l}10,59 \\
(4,25)\end{array}$ & $\begin{array}{c}12.51 \\
(3.47)\end{array}$ & $\begin{array}{l}13.66 \\
(3.74)\end{array}$ & $\begin{array}{l}13.54 \\
(3.72)\end{array}$ & $\begin{array}{l}12.83 \\
(3,05)\end{array}$ & \\
\hline R carré & & 0,46 & 0,48 & 0,55 & 0,59 & 0,59 & 0,61 & 0,63 & 0,63 & \\
\hline
\end{tabular}

Sources : tableaux 3, 4 et 5 .

abord puisque la proportion de naissances de faible poids reprêsenterait le principal déterminant de la mortalité infantile (Eberstein, Nam et Hummer, 1990; Lepage, Levasseur et Colin, 1990; McCormick, 1985; Miller, 1985). Cependant, le tableau 6 montre qu'à l'échelle des territoires de CLSC la mortalité infantile présente une association plus forte avec les caractéristiques socio-économiques qu'avec la proportion de naissances de faible poids. D'autre part, cette variable est fortement reliée à la pauvreté (Wilkins, Sherman et Best, 1991). Dans la régression multiple, l'influence de la proportion de naissances de faible poids sur la mortalité infantile une fois que le taux de pauvreté est tenu constant représente un effet 
rêsiduel qui devient difficile à prévoir. L'on ne doit donc pas s'étonner des résultats obtenus ci-dessus.

Le graphique 1 montrait plus haut que certains territoires se démarquaient distinctement de la droite de régression obtenue à partir de la relation entre la pauvreté et l'espérance de vie. Le territoire de Parc Extension présentait une espérance de vie élevée malgré une forte proportion de personnes pauvres. Or ce territoire comprend une proportion très élevée d'immigrants (58\%). Il en est de même des autres territoires qui se situalent bien au-dessus de la droite. Inversement, ceux qui apparaissaient nettement sous la droite comprenaient généralement de faibles pourcentages d'immigrants.

La proportion de pauvres et le pourcentage d'immigrants sont faiblement corrélés entre eux $(r=0,12)$. La proportion d'immigrants à elle seule présente une association relativement faible avec l'espérance de vie $(r=0,50)$ et très faible avec le taux de mortalité infantile $(r=-0,02)$. Les coefficients élevés obtenus à partir de la combinaison de ces deux variables indépendantes, particulièrement dans le cas de l'espérance de vie à la naissance, montrent leur complémentarité. L'importance des immigrants expliqueraft une bonne partie des variations de la mortalité inexpliquées par la pauvreté. Ainsi, si l'on contrôle pour la pauvreté, le coefficient de corrélation entre la proportion d'immigrants et l'espérance de vie passerait de 0,50 à 0,80 . De même, si on contrôle pour le pourcentage d'immigrants, le coefficient de corrélation entre la proportion de personnes pauvres et l'espérance de vie augmenterait de $-0,68$ à $-0,87$.

Si la relation entre le statut socio-économique et la mortalité est bien documentée, la relation entre l'immigration lou d'autres variables socio-culturelles) et la mortalité l'est beaucoup moins. Plusieurs études ont montré que les immigrants, alnsi que d'autres communautés apparentées (allophones, groupes ethniques autres que français et britannique), présenteraient une mortalité plus faible que la population hôte ou d'immigration plus ancienne (Trovato, 1990; Tremblay et Bourbeau, 1985; Termote et Gauvreau, 1988; Charbonneau et Maheu, 1973) ${ }^{3}$.

Trovato identifie dans la littérature plusieurs hypothèses reliées à la sous-mortalité des populations immigrantes : 
prédispositions génétiques, expositions à des facteurs de risque, sélection positive des immigrants. Dans ce dernier cas, l'on peut penser qu'une certaine sẻlection se fait dans le pays d'origine, et que les personnes dont l'état de santé est précaire ont des probabilitês moins fortes d'émigrer. Cette sélection positive d'individus en meilleure santé a probablement lieu aussi lors de l'étude des dossiers des candidats à l'immigration, puisque les lois canadiennes concernant l'immigration sont de plus en plus restrictives. Ce processus de sélection pourrait expliquer en partie la mortalité relativement faible de certains territoires prêsentant des proportions élevées d'immigrants.

La proportion d'immigrants a un impact moins important sur les variations géographiques de la mortalitê infantile que sur celles de l'espérance de vie à la naissance. Le taux de mortalité infantile ne concernant que la première année de la vie, le processus de sélection positive des immigrants jouerait un rôle moins important que pour l'espérance de vie. D'autre part, la mortalité infantile peut être influencée par la disponibilité des programmes de soins prénatals et post-natals (Miller, 1985; Eberstein. Nam et Hummer, 1990). Or les immigrants et les communautés culturelles pourraient recourir moins souvent aux services de santé que la population hôte pour des raisons d'ordre culturel et linguistique : insatisfaction, méfiance à l'endroit du réseau public, méconnaissance des services disponibles, etc. (Cousineau, $1989: 22-23$ ).

La régression permet non seulement de mesurer la forme, le sens et la force des associations entre variables, mais aussi de prêdire la valeur de la variable dépendante à partir de valeurs particulières que peuvent prendre les variables indépendantes.

Il est possible, par exemple, d'estimer l'espérance de vie à la naissance à partir de la proportion de personnes pauvres et du pourcentage d'immigrants selon l'équation de régression fournie à la colonne 2 du tableau 7 :

Espérance de vie $=77,8-(0,17 * \%$ Pauvres $)+(0,12 * \%$ Immigrants $)$.

Cette équation expliquant $82 \%$ des variations de l'espérance de vie, les valeurs estimées devraient être assez près des valeurs observées. Le même exercice peut être fait pour la période 19791983, à partir de l'équation :

Espérance de vie $=76,8-(0,19 * \%$ Pauvres $)+(0,12 * \%$ Immigrants $)$.

Dans ce dernier cas, le coefficient de dêtermination est de 0,83. 
Pour l'ensemble de la région, l'écart entre les espérances de vie estimées et observées est nul pour la période 1984-1988 et de seulement 0,1 an pour 1979-1983 (tableau 9). À l'échelle des territoires de CLSC, les écarts sont plus importants mais atteignent plus de trois ans dans seulement un cas (Saint-Louis du Parc, 1979-1983). Pour la période 1984-1988, dix-sept territoires enregistrent un écart égal ou supérieur à un an entre les valeurs observêes et estimées, mais la différence dépasse deux ans dans seulement cinq cas. L'on observe des résultats semblables pour la période précédente.

Les données se rapportant aux proportions de pauvres et d'immigrants permettent donc de prédire assez bien l'espérance de vie, tant pour la période 1984-1988 que pour la période antérieure. La précision est cependant variable d'un territoire à l'autre. D'autre part, comme l'espérance de vie ne présente pas de variations importantes entre plusieurs territoires, les valeurs estimées ne sont souvent pas assez précises pour permettre d'identifier les disparités géographiques.

Il serait intéressant de voir si cette méthode peut être utilisée pour estimer l'espérance de vie de territoires plus petits (secteurs de recensement), ou de territoires situés à l'extérieur de la région de Montréal. Pour les CLSC localisés hors de la région montréalaise, nous avons examiné de façon exploratoire les associations entre l'espérance de vie et certaines caractéristiques socio-êconomiques (proportions de pauvres, de familles monoparentales, d'immigrants). Les résultats obtenus ont montré une très faible association entre l'espérance de vie et ces caractéristiques de la population. L'on ne retrouve donc pas à l'extérieur de Montréal les relations observées dans la région montréalaise.

\section{CONCLUSION}

Malgré une baisse importante des indices de mortalité dans la région de Montréal, l'on retrouve toujours à la fin des années 1980 des disparités géographiques importantes. Un écart de plus de dix ans en espérance de vie est observé entre certains territoires, et cet écart n'a pas diminué pendant les dix dernières années.

La proportion de personnes pauvres et le pourcentage d'immigrants combinés ensemble expliqueraient plus de $80 \%$ des variations géographiques de l'espérance de vie, mais seu- 
TABLEAU 9

Espérances de vie à la naissance observées et estimées, CLSC de la région de Montréal, 1979-1983 et 1984-1988 (en années)

\begin{tabular}{|c|c|c|c|c|c|c|c|c|}
\hline \multirow[b]{3}{*}{ Territoires } & \multicolumn{4}{|c|}{$1979-1983$} & \multicolumn{4}{|c|}{$1984-1988$} \\
\hline & \multicolumn{2}{|c|}{ Espér. de vie } & \multicolumn{2}{|c|}{ Écart } & \multicolumn{2}{|c|}{ Espér. de vie } & \multicolumn{2}{|c|}{ Écart } \\
\hline & Obs. & Est. & Abs. & $\begin{array}{l}\text { Rel. } \\
(\%)\end{array}$ & Obs. & Est. & Abs. & $\begin{array}{l}\text { Rel. } \\
(\%)\end{array}$ \\
\hline 11 - Lac St-Louis & 78.7 & 78,2 & -0.5 & $-0,7$ & 79,6 & 79,3 & $-0,3$ & $-0,4$ \\
\hline 13 - Pierrefonds & 75,8 & 77,0 & 1,2 & 1,6 & 75,6 & 77,9 & 2,3 & 3,1 \\
\hline 14 - Dollard-des-Ormeaux & 77,2 & 78.6 & 1,4 & 1,8 & 78.5 & 79,8 & 1.3 & 1,6 \\
\hline 15 - Vieux Lachine & $\mathbf{7 4 . 9}$ & 74,9 & -0.0 & $-0,0$ & 75,6 & 76,0 & 0,4 & 0,5 \\
\hline 21 - Pointe St-Charles & 68,9 & 68,6 & $-0,3$ & $-0,4$ & 69,8 & 9,8 & $-0,0$ & $-0,0$ \\
\hline 22 - Verdun & 72,9 & 72,8 & $-0,1$ & $-0,2$ & 74.9 & 73.9 & -1.0 & $-1,3$ \\
\hline 24 - Ville-Émard-Côtc St-Paul & 74.6 & 73.7 & $-0,9$ & $-1,1$ & 75,1 & $\mathbf{7 4 , 4}$ & -0.7 & $-1,0$ \\
\hline 26 - Lasalle & 75,2 & 75,9 & 0,7 & 0,9 & 76,5 & 76,9 & 0,4 & 0,6 \\
\hline 31 - Rivière-des-Prairies & 78,5 & 75,8 & $-2,6$ & $-3,4$ & 9.0 & 77,8 & -1.2 & $-1,5$ \\
\hline 32 - Octave-Roussin & 71.4 & 73.7 & 2,4 & $\mathbf{3 , 4}$ & $\mathbf{7 4 , 0}$ & 75.1 & 1,1 & 1,4 \\
\hline 33 - Mercier-Est & 73,0 & 74,0 & 1,1 & 1.5 & 73,9 & 74,9 & 1,0 & 1.4 \\
\hline 34-Olivi & 74,0 & $\mathbf{7 4 , 9}$ & 0,8 & 1.1 & $\mathbf{7 4 , 8}$ & 5,9 & 1,1 & 1,4 \\
\hline 35 - Hochelaga-N & 71,4 & 70.7 & -0.8 & -1.1 & 72.3 & 71.8 & -0.5 & -0.7 \\
\hline 36 - Rosemont & 74,6 & 73,7 & $-0,9$ & $-1,2$ & 75,5 & $\mathbf{7 4 , 5}$ & $-1,0$ & $-1,3$ \\
\hline 38 - Anjou & 77,3 & 75,3 & $-2,0$ & $-2,6$ & 77,6 & 76,3 & $-1,3$ & $-1,7$ \\
\hline 39 - St-Léo & 77,2 & 77,6 & 0,3 & 0,4 & 78,5 & 78,4 & -0.1 & $-0,2$ \\
\hline 41 -Cóte & 79,1 & 77.0 & -2.1 & -2.7 & 80.1 & 77.3 & -2.8 & $-3,5$ \\
\hline 42 - Snowdon & 78.0 & 77,6 & $-0,4$ & $-0,5$ & $\mathbf{7 7 . 5}$ & $\mathbf{7 8 , 8}$ & 1,3 & 1,7 \\
\hline 43 - Côte-St-Luc-Hampstead & $\mathbf{7 8 , 4}$ & 78,8 & 0,3 & 0,4 & 79,5 & 79,8 & 0,3 & 0,3 \\
\hline 44 - Outremont-Mont-Royal & 79,9 & 77,6 & $-2,3$ & $-2,8$ & 81.5 & 78,8 & $-2,7$ & $-3,3$ \\
\hline $51-\mathrm{NDG}$ & 76.1 & 76.8 & 0.7 & 0.9 & 77.7 & 77,7 & 0,0 & 0,0 \\
\hline 52 - Métro ( & 77.8 & 78,3 & 0,5 & 0,7 & 80,0 & 79.4 & -0.6 & -0.7 \\
\hline Sud-Ouest) & 74,0 & 75,9 & 2,0 & 2,7 & $\mathbf{7 4 , 1}$ & 76,7 & 2,6 & 3.5 \\
\hline 54 - St-Louis du Parc & 70.9 & $\mathbf{7 4 . 2}$ & 3,3 & 4.7 & 73.1 & 75,5 & 2,4 & 3,3 \\
\hline 55 - St-Henri- & 69,4 & 69,2 & $-0,2$ & $-0,2$ & 71,1 & 71,6 & 0,5 & 0.7 \\
\hline 61 - Montré & 74,8 & 74,2 & $-0,6$ & -0.8 & 75.4 & 75.1 & -0.3 & -0.4 \\
\hline 63 - St-Michel & 75.9 & 75,0 & $-0,8$ & $-1,1$ & 76,2 & 75,8 & $-0,4$ & $-0,5$ \\
\hline 65 - Ahuntsic & 75,7 & 75,4 & $-0,3$ & $-0,4$ & 76,7 & 76,4 & $-0,3$ & $-0,4$ \\
\hline 66 - Bordeaux-Cartierville & 76,9 & 76,9 & 0.0 & 0,0 & 77.9 & 78,1 & 0.2 & 0.3 \\
\hline 68 - St-Laurent & 76,7 & 77,6 & 0,8 & 1.1 & 78,3 & 78,3 & 0.0 & 0.0 \\
\hline 71 - Centr & 68,5 & 68,7 & 0.1 & 0,2 & 70,6 & 70,9 & 0,3 & 0,4 \\
\hline 72 - Plate & 70,1 & 71,2 & 1,1 & 1.5 & 71,6 & 72.4 & 0,8 & 1,1 \\
\hline 74 - Parc Extension & 77.2 & 76,4 & -0.8 & $-1,1$ & 77.6 & 76.6 & $-1,0$ & -1.3 \\
\hline 75 - Centre-Ville & 68,6 & 69.0 & 0.4 & 0.6 & 72,3 & 72,8 & 0.5 & 0.7 \\
\hline 76 - Villeray & 75,3 & 74,1 & $-1,2$ & $-1,6$ & 76,0 & 75,1 & $-0,9$ & $-1,2$ \\
\hline 77 - Petite Patrle & 73,3 & 72,3 & $-1,0$ & $-1,4$ & 74,5 & 73,4 & -1.1 & -1.5 \\
\hline 81 -Des Mille-Îles & 75,6 & 75,9 & 0,3 & 0.4 & 77,2 & 76,7 & -0.5 & $-0,6$ \\
\hline 83 - Norman-Bethune & 75,5 & 75,9 & 0.4 & 0.5 & 76,8 & 77,2 & 0,4 & 0,5 \\
\hline 85 - Du Marigot & 75,7 & 74,8 & $-0,9$ & $-1,2$ & 77,1 & 75,8 & $-1,3$ & $-1,7$ \\
\hline 87 - Ste-Rose de Lava & 74,2 & 75.1 & 0.9 & 1.2 & 75.2 & 76.6 & 1.4 & 1.9 \\
\hline Région de Montréal & 75,0 & 75,1 & 0.1 & 0,2 & 76,2 & 76,2 & 0,0 & 0,0 \\
\hline
\end{tabular}

Sources : tableaux 3 et 4; Wilkins, 1987. 
lement $55 \%$ des variations de la mortalité infantile lorsque l'on tient compte en plus, dans ce dernier cas, de la proportion des naissances de faible poids.

L'approche écologique utilisée ne permet pas d'établir des liens entre les caractéristiques de la population et la mortalité à l'échelle individuelle. Les résultats obtenus dans cette étude ne nous permettent pas de conclure directement que les personnes pauvres ou les immigrants présentent des niveaux de mortalité différents de ceux du reste de la population. Nous croyons cependant que les relations observêes à l'êchelle des CLSC sont un reflet des inégalités sociales qui existent dans la population.

Cette étude porte sur la mortalité générale. Il serait intéressant d'effectuer le même exercice pour des causes de décès spécifiques et pour la morbidité hospitalière.

Si l'approche écologique est souvent utilisée dans l'étude des relations entre la mortalité et le niveau socio-économique, c'est que les actes de décès ne contiennent pas de variables permettant d'identifier le niveau socio-êconomique de l'individu. Il serait possible de contourner ce problème, dans le cas par exemple de la mortalité infantile, en jumelant les informations provenant du fichter des naissances à celles fournies par le fichier des décès à partir de certaines variables apparaissant à la fois sur les deux fichiers (date de naissance, nom de la mère, etc.). L'on pourrait ainsi calculer des taux de mortalité infantile selon le niveau de scolarité de la mère apparaissant sur l'acte de naissance. De la même façon, il serait possible d'étudier la mortalité infantile selon le poids à la naissance et la durée de gestation. Les résultats obtenus seraient beaucoup plus précis que ceux provenant des études écologiques et permettraient de mieux identifier les facteurs en cause dans les variations de la mortalitê. 


\section{REFFÉRENCES BIBLIOGRAPHIOUES}

BERRY, William D., et Stanley FELDMAN, 1990. Multiple Regression in Practice. Beverly Hills, Sage Publications, 93 p.

BUREAU DE LA STATISTIQUE DU QUÉBEC, 1972. Tables de mortalité abrégées. Régions administratives, 1950-52, 1955-57, 1960-62 et 1965-67. Quêbec, Gouvernement du Québec, 195 p.

BUREAU DE LA STATISTIQUE DU QUÉBEC, 1988. La situation démographique au Québec. Édition 1988. Québec, Les Publications du Quêbec, $198 \mathrm{p}$.

CHARBONNEAU, Hubert, et Robert MAHEU, 1973. Les aspects démographiques de la question linguistique. Synthèses réalisées pour le compte de la Commission d'enquête sur la situation de la langue française et sur les droits linguistiques au Québec, no 3. Quêbec, Editeur officiel du Québec, 438 p.

CHOINIĖRE, Robert, 1990a. La situation démographique et soctoéconomique de la population du Montréal métropolitain : princtpaux résultats du recensement de 1986 par CLSC et DSC. Montréal, Regroupement de départements de santé communautaire, $196 \mathrm{p}$.

CHOINIËRE, Robert, 1990b. Dossier socio-démographique et sanitatre du territoire du Département de santé communautaire de l'Hôpital général de Montréal. Hôpital général de Montréal, Département de santé communautaire, $193 \mathrm{p}$.

COUSINEAU, Daniel, 1989. Les communautés culturelles. Collection "Et la santé, ça va ?», no 5. Québec, Les Publications du Québec, $54 \mathrm{p}$.

EBERSTEIN, Isaac, Charles B. NAM et Robert A. HUMMER, 1990. "Infant Mortality by Cause of Death: Main and Interaction Effects". Demography, 27, 1, 413-430.

FLEISS, Joseph L., 1981. Statistical Methods for Rates and Proportions. New York, John Wiley and Sons, Inc. 321 p.

GUILLEMETTE, André, 1983. «L'évolution de la mortalité différentielle selon le statut socio-économique sur l'île de Montréal.. Cahiers québécois de démographie, 12, 1, 29-50.

HOEY, John, Russell WILKINS, Gilbert GAGNON et Jennifer O'LOUGHLIN, 1987. L'état de santé des Québécois : un profll par régton socio-sanitaire et par département de santé communautaire. Commission d'enquête sur les services de santé et les services sociaux, document de recherche no 11. Québec, Les Publications du Québec, $96 \mathrm{p}$.

KLEINBAUM, David G., Lawrence L. KUPPER et Keith E. MULLER, 1987. Applied Regression Analysis and Other Multivariable Methods. Boston, PWS-Kent Publishing Company, 718 p.

LANGBEIN, Laura I., et Allan J. LICHTMAN, 1988. Ecological Inference. Beverly Hills, Sage Publications, $70 \mathrm{p}$.

LAST, John M., 1988. A Dictionary of Epidemiology. New York, Oxford University Press, 2e édition, 141 p. 
LEPAGE, M. C., M. LEVASSEUR et C. COLIN, 1990. Prévention de l'insuffisance de poids à la naissance. Problématique et plan d'action. Présenté au Comité Famille-Enfance. Division santé communautaire de l'Association des hôpitaux de Guébec.

LEWIS-BECK, Michael S., 1990. Applied Regression. An Introduction. Beverly Hills, Sage Publications, 79 p.

McCORMICK, M., 1985. "The Contribution of Low Birth Weight to Infant Mortality and Childhood Morbidity". The New England Journal of Medicine, 312, 2, 82-90.

MILLER, C. Arden, 1985. "Infant Mortality in the U.S.». Scientific American, 253, 1, 31-39.

MINISTĖRE DES AFFAIRES SOCIALES DU QUÉBEC, 1970. Rapport annuel 1969 et 1970. Registre de la population. Québec, Gouvernement du Québec, $195 \mathrm{p}$.

MONNIER, Alain, 1988. "La conjoncture démographique : l'Europe et les pays développés d'outre-mer". Population, 4-5, 893-904.

MORGENSTERN, Hal, 1982. «Uses of Ecological Analysis in Epidemiological Research*. American Journal of Public Health, 72, 12, 1336-1344.

O'LOUGHLIN, Jennifer, et Jean-François BOIVIN, 1987. Indicateurs de santé, facteurs de risque liés au mode de vie et utilisation du système de soins dans la région centre-ouest de Montréal. Commission d'enquête sur les services de santé et les services soclaux, Synthèse critique 12. Quêbec, Les Publications du Québec, $115 \mathrm{p}$.

PÉRON, Yves, et Claude STROHMENGER, 1985. Indices démographiques et indicateurs de santé des populations. Présentation et interprétation. Ottawa, Statistique Canada, Division de la santé, $265 \mathrm{p}$.

ROTHMAN, Kenneth J., 1986. Modern Epidemiology. Boston, Little, Brown and Company, $358 \mathrm{p}$.

SCHROEDER, Larry, David L. SJOgUIST et Paula E. STEPHAN, 1989. Understanding Regression Analysis. An Introductory Guide. Beverly Hills, Sage Publications, 95 p.

STATISTIGUE CANADA, 1988. Naissances et décès : la statistique de l'état civil. Volume 1, 1986. Ottawa, catalogue 84-203, 14-15.

STATISTIQUE CANADA, 1990. Tables de mortalité, Canada et provinces, 1985-1987. Ottawa, Centre canadien d'information sur la santé, catalogue 82-0035, "Rapports sur la santë", supplément no 13, vol. 2, no 2, 56 p.

TREMBLAY, Marc, et Robert BOURBEAU, 1985. „La mortalité et la féconditê selon le groupe linguistique au Quêbec, 1976 et 1981". Cahiers québécois de démographie, 14, 1, 7-30.

TERMOTE, Marc, et Danielle GAUVREAU, 1988. La situation démolinguistique du Québec. Conseil de la langue française. Québec, Éditeur officiel du Québec, 292 p.

TROVATO, Frank, 1990. "Immigrant Mortality Trends and Differentials". Dans S. SHIVA, F. TROVATO et L. DRIEDGER, éd. 
Ethnic Demography. Canadian Immigrant, Racial and Cultural Variations. Ottawa, Carleton University Press, 91-110.

WILKINS, Russell, 1979. L'espérance de vie par quartier à Montréal, 1976 : un indicateur social pour la planification. Montréal, Institut de recherches politiques, $35 \mathrm{p}$.

WILKINS, Russell, 1980a. "L'inégalité sociale face à la mortalité à Montréal, 1975-1977^. Cahiers québécois de démographie, 9, 2, 157-184.

WILKINS, Russell, 1980b. L'état de santé au Canada, 1926-1976. Montréal, Institut de recherches politiques, $36 \mathrm{p}$. (document hors série no 13).

WILKINS, Russell, 1980c. "Differential mortality in Montreal, 19611976». Communication présentée au colloque annuel de la Canadian Population Society (inédit).

WILKINS, Russell, 1987. Dossier population: compilations du recensement de 1981 par CLSC et DSC, région 6A (Montréal-métropolitain). Montréal, Regroupement de départements de santé communautaire du Montréal métropolitain.

WILKINS, Russell, et Owen ADAMS, 1983. Healthfulness of Life: A Unified Vlew of Mortality. Institutionalization, and Noninstitutionalized Disability in Canada, 1978. Montréal. Institut de recherches politiques, $153 \mathrm{p}$.

WILKINS, Russell, Gregory J. SHERMAN et P. A. F. BEST, 1991. Issues de grossesse et mortalité infantile selon le revenu dans les régions urbaines du Canada en 1986. Ottawa, Statistique Canada, kRapports sur la santé,, 3, 1, 7-34, catalogue 82-003.

WILKINS, Russell, Owen ADAMS et Anna BRANCKER, 1989. Évolution de la mortalité selon le revenu dans les régions urbaines entre 1971 et 1986. "Rapports sur la santéw, 1, 2, 137-174. 


\section{RESUME - SUMMARY - RESUMEN}

CHOINIERE Robert - LES DISPARITES GEOGRAPHIQUES DE LA MORTALITE DANS LE MONTREAL METROPOLITAIN, 1984-1988: ETUDE ECOLOGIQUE DES LIENS AVEC LES CONDITIONS SOCIALES, ECONOMIQUES ET CULTURELLES

Malgré une baisse notable des indices de mortalité dans la région de Montréal, l'on retrouve toujours à la fin des années 1980 des disparités géographiques importantes. Un écart de plus de dix ans en espérance de vie est observé entre certains quartiers, et cet écart n'a pas diminué durant les dix dernières années. L'étude écologique des liens entre la mortalité et diffërentes caractêristiques de la population à partir des méthodes de la régression linéaire montre que la proportion de personnes pauvres et le pourcentage d'immigrants combinés expliqueraient ensemble plus de $80 \%$ des variations géographiques de l'espérance de vie à Montréal.

CHOINIËRE Robert - SPATIAL MORTALITY DISPARITIES IN THE METROPOLITAN REGION OF MONTREAL, 1984-1988: AN ECOLOGICAL ANALYSIS OF THE ROLE OF SOCLAL, ECONOMIC AND CULTURAL FACTORS

Despite a marked drop of mortality in the Montreal region, there are still important geographic disparities at the end of the eighties. There is a difference of more than ten years in life expectancy between some areas, and this has not changed over the last decade. A multiple regression analysis shows that the proportion of poors and the percentage of immigrants account for more than $80 \%$ in the geographic disparities of life expectancy in the Montreal region.

CHOINIERE Robert - DESNIVELES GEOGRAFICOS DE MORTALDAD EN LA REGION METROPOLITANA DE MONTREAL, 1984-1988: ANALISIS ECOLOGICO DE LA FUNCION DE LAS CONDICIONES SOCIALES, ECONOMICAS Y CULTURALES

A pesar de la disminución notable de los índices de mortalidad en la región de Montréal, se encuentran todavia, a fines de los años ochentas, desniveles geográficos importantes. Se observa una diferencia de más de diez años de la esperanza de vida en clertos barrios, diferencia que no ha disminuido en los ültimos diez años. El análisis de regresión lineal de la relación entre la mortalidad y distintas caracteristicas de la población demuestra que la proporción de personas pobres $y$ el porcentaje de inmigrantes representan más del $80 \%$ de las variaciones geográficas de la esperanza de vida en Montréal. 